\title{
The eccentric massive binary V380 Cyg: revised orbital elements and interpretation of the intrinsic variability of the primary component ${ }^{\star}$
}

\author{
A. Tkachenko, ${ }^{1} \dagger \ddagger$ P. Degroote, ${ }^{1} \S$ C. Aerts,${ }^{1,2}$ K. Pavlovski, ${ }^{3}$ J. Southworth, ${ }^{4}$ \\ P. I. Pápics, ${ }^{1}$ E. Moravveji, ${ }^{1}$ V. Kolbas, ${ }^{3}$ V. Tsymbal,${ }^{5}$ J. Debosscher ${ }^{1}$ and K. Clémer ${ }^{1}$ \\ ${ }^{1}$ Instituut voor Sterrenkunde, KU Leuven, Celestijnenlaan 200D, B-3001 Leuven, Belgium \\ ${ }^{2}$ Department of Astrophysics, IMAPP, Radboud University Nijmegen, NL-6500 GL Nijmegen, the Netherlands \\ ${ }^{3}$ Department of Physics, University of Zagreb, Bijenička cesta 32, 10000 Zagreb, Croatia \\ ${ }^{4}$ Astrophysics Group, Keele University, Staffordshire ST5 5BG, UK \\ ${ }^{5}$ Department of Astronomy, Tavrian National University, Simferopol, Ukraine
}

Accepted 2013 December 12. Received 2013 December 12; in original form 2013 October 27

\begin{abstract}
We present a detailed analysis and interpretation of the high-mass binary V380 Cyg, based on high-precision space photometry gathered with the Kepler space mission as well as highresolution ground-based spectroscopy obtained with the HERMES spectrograph attached to the $1.2 \mathrm{~m}$ Mercator telescope. We derive a precise orbital solution and the full physical properties of the system, including dynamical component mass estimates of $11.43 \pm 0.19$ and $7.00 \pm 0.14 \mathrm{M}_{\odot}$ for the primary and secondary, respectively. Our frequency analysis reveals the rotation frequency of the primary in both the photometric and spectroscopic data and additional low-amplitude stochastic variability at low frequency in the space photometry with characteristics that are compatible with recent theoretical predictions for gravity-mode oscillations excited either by the convective core or by sub-surface convective layers. Doppler imaging analysis of the silicon lines of the primary suggests the presence of two high-contrast stellar surface abundance spots which are located either at the same latitude or longitude. Comparison of the observed properties of the binary with present-day single-star evolutionary models shows that the latter are inadequate and lack a serious amount of near-core mixing.
\end{abstract}

Key words: binaries: eclipsing-stars: fundamental parameters-stars: individual: V380 Cyg - stars: oscillations - stars: variables: general.

\section{INTRODUCTION}

V380 Cyg (HR 7567, HD 187879, KIC 5385723) is a bright $(V=5.68)$ double-lined spectroscopic binary (SB2) consisting of two B-type stars residing in an eccentric $12.4 \mathrm{~d}$ orbit. The primary

\footnotetext{
${ }^{\star}$ Based on the data gathered with NASA's Discovery mission Kepler and with the HERMES spectrograph, installed at the Mercator telescope, operated on the island of La Palma by the Flemish Community, at the Spanish Observatorio del Roque de los Muchachos of the Instituto de Astrofísica de Canarias and supported by the Fund for Scientific Research of Flanders (FWO), Belgium, the Research Council of K. U. Leuven, Belgium, the Fonds National de la Recherche Scientific (F.R.S.-FNRS), Belgium, the Royal Observatory of Belgium, the Observatoire de Genève, Switzerland and the Thüringer Landessternwarte Tautenburg, Germany.

$\dagger$ E-mail: andrew@ ster.kuleuven.be

$\ddagger$ Postdoctoral Fellow of the Fund for Scientific Research (FWO), Flanders, Belgium.

$\S$ Postdoctoral Fellow of the Fund for Scientific Research (FWO), Flanders, Belgium.
}

component is an evolved star which is about to start its hydrogen shell burning phase, whereas the secondary, fainter component is a main-sequence star comparable in temperature. Most of the system's brightness comes from the primary, with the secondary contributing about 6 per cent of the total flux. For this reason, the system was thought to be a single-lined spectroscopic binary for a long time until Batten (1962) reported the detection of the secondary's spectral lines on 22 newly obtained spectrograms. Although the double-lined nature of the star was a subject of debate for another 20 years (see e.g. Popper 1981), Hill \& Batten (1984) confirm that V380 Cyg is an SB2 star based on newly obtained high-dispersion spectroscopic data. In addition to the orbital elements which differ insignificantly from those derived by Batten (1962), the authors report on apsidal motion of about 0.011 per period.

Since then, V380 Cyg was the subject of several more extensive studies based on both ground-based spectroscopy and multicolour photometry. Guinan et al. (2000, hereafter G2000) combined newly obtained differential $U, B, V$ photometry with the spectroscopic data obtained by Popper \& Guinan (1998) to refine the orbital solution and to determine precise physical properties of both binary 
components. The times of light minima were used to measure apsidal motion of the system which was found to be of $24^{\circ}$ per 100 years ( 0.008 per period), slightly lower than but in reasonable agreement with the value reported by Batten (1962).

Eclipsing binary stars are a prime source for testing evolutionary models, as their fundamental parameters like masses, radii, etc. can be derived to a very high precision. The proper solution of the convective energy transport is one of the biggest issues of the current evolutionary models. However, comparison of the observed stellar properties with the theoretical predictions allows the deduction of an important parameter such as the convective overshooting $\alpha_{\mathrm{ov}}$ (that stands for the stellar convective core overextension). For V380 Cyg, G2000 found that the position of the more massive primary on the Hertzsprung-Russell (HR) diagram requires an extremely high value of $\alpha_{\text {ov }}$ of about $0.6 \pm 0.1$ to match the observed properties of the star with the theoretical models. This is actually the highest known value of $\alpha_{\text {ov }}$ for B-type stars, whereas the typical value is of the order of 0.2 for single stars with similar masses (see e.g. Aerts et al. 2003, 2011; Briquet et al. 2011). Assuming this typical value of $\alpha_{\mathrm{ov}}$ for the primary of V380 Cyg leads to inconsistency between the mass deduced from the binary dynamics and the one obtained from the evolutionary models. This inconsistency between the two masses is usually called the mass discrepancy. It is worth noting that the second highest value after V380 Cyg of $\alpha_{\mathrm{ov}} \sim 0.45$ has also been found for the $\sim 8 \mathrm{M}_{\odot}$ primary component of a detached binary system (Briquet et al. 2007). Alternatively, the rate of the apsidal motion, depending on the internal mass distribution of a star, can be used to estimate the overshooting parameter $\alpha_{\text {ov }}$. The value obtained by G2000 in this way perfectly agrees with the one deduced from the position of the star in the HR diagram. On the other hand, Claret (2007) found that the apsidal motion rate of the V380 Cyg is compatible with $\alpha_{\text {ov }}=0.4_{-0.3}^{+0.2}$.

Pavlovski et al. (2009, hereafter P2009) collected about 150 highresolution échelle spectra using several telescopes and performed a detailed spectroscopic study of the system with the aim to determine the precise orbital solution as well as the physical parameters of both stellar components. The authors also revisited the $U, B, V$ light curves obtained by G2000 and analysed the data using a modified version of the Wilson-Devinney (WD) code (Wilson \& Devinney 1971; Wilson 1979, 1993). The spectral disentangling (SPD) technique applied by P2009 to their spectra, besides providing precise orbital elements, revealed a faint secondary component in the composite spectra and provided high-quality disentangled spectra for both stars. The spectra were further analysed to determine the fundamental parameters as well as to do the abundance analysis for the primary. We refer to Table 1 for an intercomparison between the physical parameters of the stars derived by G2000 and P2009.

Similar to G2000, P2009 found that the position of the more massive primary in the HR diagram does not match the theoretical predictions for a single star of a similar mass. The authors find a mass discrepancy of about $1.5 \mathrm{M}_{\odot}$ for the primary component of V380 Cyg. Though this is much less than what was found for V380 Cyg by, e.g., Lyubimkov et al. (1996) who reported a mass discrepancy of 3.4 and $1.1 \mathrm{M}_{\odot}$ for the primary and the secondary, respectively, the value is still significant. The mass discrepancy problem observed in massive O- and B-type stars has been known for more than 20 years already and has been discussed in detail by Herrero et al. (1992). Hilditch (2004) pointed out that the discrepancy does not disappear when the effects of rotation are included into the models; P2009 came to the same conclusion concerning the primary of V380 Cyg.
It is thus important to have an independent estimate of the core overshooting parameter $\alpha_{\mathrm{ov}}$ for this type of stars, and this is provided by the asteroseismic methods. In Tkachenko et al. (2012, hereafter Paper I), we presented results of the preliminary analysis of our data consisting of high-quality Kepler space photometry and highresolution, ground-based spectroscopy. We confirmed the doublelined nature of the binary and presented precise orbital elements, in general agreement with the two previous findings by G2000 and P2009. Both photometric and spectroscopic data revealed the presence of variability intrinsic to the primary component, which we interpreted in terms of stochastically excited gravity (g-) mode oscillations. Moreover, both data sets showed that at least part of this variability occurs at (higher) harmonics of the orbital frequency suggesting a tidal nature for the pulsations.

In this paper, we present a solid interpretation of our full data set that consists of the data presented in Paper I and additional four quarters of Kepler data and some 50 high-dispersion, high signalto-noise ratio $(\mathrm{S} / \mathrm{N})$ spectra. The data themselves as well as the data reduction procedure are described in Section 2. Photometric analysis of the system is presented in Section 3 while Section 4 is devoted to the analysis of our spectra. Discussion and conclusions are presented in Section 5 .

\section{OBSERVATIONS AND DATA REDUCTION}

The photometric analysis of the V380 Cyg system presented in this paper is based on the high-quality Kepler space data. The data are released in quarters (periods of time between the two consecutive spacecraft rolls needed to keep the solar shutters pointing towards the Sun); each quarter comprises about three months of nearly continuous observations. The telescope operates in two different modes, long and short cadence (LC and SC, respectively), which differ from each other by the time of integration which is $29.42 \mathrm{~min}$ for the LC and $58.85 \mathrm{~s}$ for the SC data. The exposure time is the same in both modes and equals $6.54 \mathrm{~s}(0.52 \mathrm{~s}$ of readout included $)$. Thus, the two modes basically differ by the time resolution where the LC integrates over 270 single exposures whereas one data point in the SC mode contains nine exposures. For V380 Cyg, we use all available Kepler data, i.e. Q7, Q9-Q10 and Q12-Q14, which gives a time span of $\sim 560 \mathrm{~d}$.

Because of the extreme brightness of the system $(V=5.68)$, a dedicated customized aperture mask was defined to collect the flux from the star. This is fully justified given that Kepler has proved already that it can provide high-precision photometry even for highly saturated targets (see, e.g., Bryson et al. 2010; Kolenberg et al. 2011; Paper I).

In addition to the photometric data, high-dispersion ground-based spectroscopy has been acquired with the HERMES spectrograph (Raskin et al. 2011) attached to the $1.2 \mathrm{~m}$ Mercator telescope (La Palma, Canary Islands). All spectra have a resolving power of $\sim 85000$ and cover the wavelength range from 380 to $900 \mathrm{~nm}$. Table 2 gives the journal of observations listing the calendar and the Julian dates in its first two columns, and the mean exposure time and the number of spectra obtained during each run in columns 3 and 4, respectively. In total, we have obtained 452 spectra with an average S/N above 200, of which 406 were part of the analysis presented in Paper I. More information about the spectroscopic data and the reduction procedure can be found in Paper I. Fig. 1 shows the phase distribution of all our out-of-eclipse spectra that will be analysed in Section 4. The radial velocities (RVs) presented in this figure were computed from our final orbital solution (cf. Table 4) and are used here for illustration purposes. 
Table 1. Physical parameters of both stellar components of the V380 Cyg binary system as derived by Guinan et al. (2000) and Pavlovski et al. (2009). Parameter errors are given in parentheses in terms of last digits.

\begin{tabular}{lcccc}
\hline \multirow{2}{*}{ Parameter } & \multicolumn{2}{c}{ Primary } & \multicolumn{2}{c}{ Secondary } \\
& G2000 & P2009 & G2000 & P2009 \\
\hline Mass $\left(\mathrm{M}_{\odot}\right)$ & $11.10(50)$ & $13.13(24)$ & $6.95(25)$ & $7.78(10)$ \\
Radius $\left(\mathrm{R}_{\odot}\right)$ & $14.70(20)$ & $16.22(26)$ & $3.74(07)$ & $4.06(08)$ \\
$\log \left(L / \mathrm{L}_{\odot}\right)$ & $4.60(03)$ & $4.73(03)$ & $3.35(04)$ & $3.51(04)$ \\
$T_{\text {eff }}(\mathrm{K})$ & $21350(400)$ & $21750(280)$ & $20500(500)$ & $21600(550)$ \\
$\log g(\mathrm{dex})$ & $3.148(023)$ & $3.136(014)$ & $4.133(023)$ & $4.112(017)$ \\
$M_{\text {bol }}(\mathrm{mag})$ & $-6.75(07)$ & $-7.06(06)$ & $-3.62(10)$ & $-4.03(10)$ \\
$V_{\text {eq }}\left(\mathrm{km} \mathrm{s}^{-1}\right)$ & $98(4)$ & $98(2)$ & $32(6)$ & $43(4)$ \\
\hline
\end{tabular}

Table 2. List of the spectroscopic observations of V380 Cyg. $\mathrm{JD}$ is the Julian date, exposure time stands for the mean value for each run and $N$ gives the number of spectra taken during the corresponding period.

\begin{tabular}{|c|c|c|c|}
\hline \multicolumn{2}{|c|}{ Time period } & \multirow{2}{*}{$\begin{array}{l}\text { Exposure time } \\
\text { (s) }\end{array}$} & \multirow[t]{2}{*}{$N$} \\
\hline Calendar date & JD-2450000 & & \\
\hline 05.04-07.04.2011 & $5656.72-5658.76$ & 952.67 & \\
\hline 09.04-16.04.2011 & $5660.65-5667.74$ & 894.28 & \\
\hline 19.04-20.04.2011 & $5670.64-5671.70$ & 853.85 & 1 \\
\hline $03.05-16.05 .2011$ & $5684.59-5697.70$ & 1100.00 & 6 \\
\hline $21.05-25.05 .2011$ & $5702.55-5706.58$ & 879.73 & 2 \\
\hline $28.05-30.05 .2011$ & $5710.49-5711.69$ & 900.00 & 1 \\
\hline $02.06-06.06 .2011$ & $5714.53-5718.72$ & 1200.00 & 21 \\
\hline $12.06-13.06 .2011$ & $5724.52-5725.72$ & 1200.00 & 20 \\
\hline 02.07-03.07.2011 & $5744.55-5744.74$ & 1200.00 & 14 \\
\hline 06.07-07.07.2011 & $5749.38-5749.74$ & 1380.95 & 21 \\
\hline $15.07-26.07 .2011$ & $5758.41-5769.41$ & 1199.73 & 72 \\
\hline 31.07-01.08.2011 & 5774.39-5774.74 & 1200.00 & 23 \\
\hline 08.08-09.08.2011 & 5782.39-5782.67 & 1200.00 & 2 \\
\hline $20.08-25.08 .2011$ & $5794.35-5798.66$ & 1200.00 & 52 \\
\hline $02.08-04.08 .2012$ & $6142.40-6143.71$ & 1223.48 & 46 \\
\hline
\end{tabular}

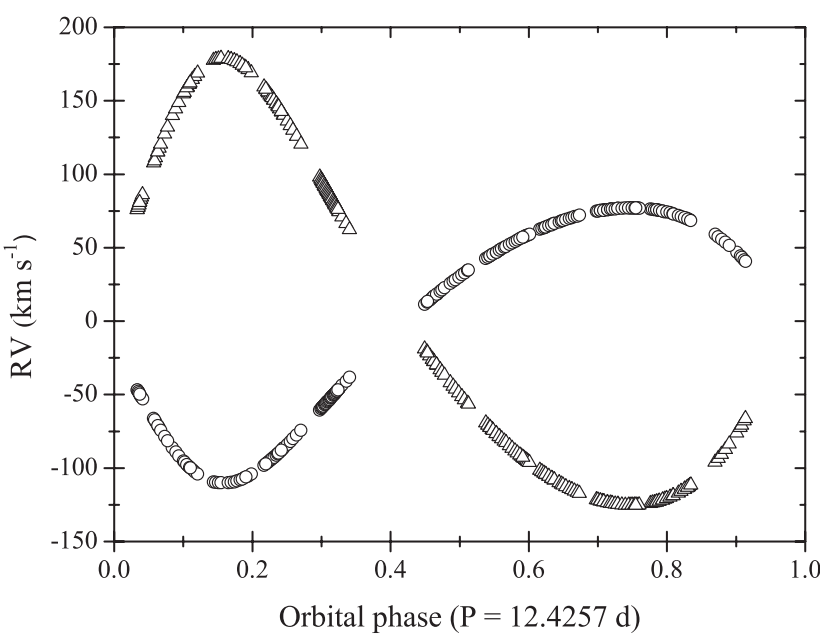

Figure 1. Orbital phase distribution of out-of-eclipse spectra in terms of the radial velocities (RVs) computed from our final orbital solution (see Section 4.1, Table 4). The primary and secondary RVs are shown by circles and triangles, respectively. Phase zero corresponds to the periastron passage.

\section{KEPLER PHOTOMETRY}

In this section, we describe the procedure used to obtain a photometric orbital solution as well as the results of the frequency analysis of the residuals computed after subtracting our best-fitting binary model from the original data.

\subsection{Orbital solution}

The effects of binarity in the light curves were modelled using the WD code (Wilson \& Devinney 1971; Wilson 1979). We used the JKTWD wrapper (Southworth et al. 2011) to drive automatic iterations of the 2004 version of the code (hereafter called wD2004).

The data were first reduced to a more manageable quantity by phase binning. This comprised converting the timestamps into orbital phase using the ephemeris of G2000, sorting in phase, and then binning them into 200 normal points. The bin sizes were chosen to be five times smaller during the eclipses, in order to provide a finer sampling of the light variations at these important times. This binning process was performed for both the combined data and for the six individual quarters, and was fundamental to making the problem tractable. The WD code becomes very slow when considering either orbital eccentricity or a high numerical accuracy; both are required for the current data. Even after binning the data to 200 normal points, it took $20-30 \mathrm{~h}$ of CPU time to fit a single light curve.

Our analysis in the current work was guided by that of the Q7 data in Paper I. Multiple alternative solutions were calculated, but our final numbers rest on the following characteristics (see Table 3). The detailed treatment of reflection in wD2004 was used, with two reflections, and the numerical grid size set to 60 for both the coarse and the fine grids. The stars were specified to rotate at 1.5 and 2 times their pseudo-synchronous velocities. The gravity darkening coefficients and albedos were set to unity and third light was fixed at zero. A linear limb darkening (LD) law was specified, and the bolometric coefficients for both stars plus the coefficient for star B were fixed to values obtained from interpolation within the tables of van Hamme (1993).

The fits were performed in Mode 2, in which the effective temperatures and passband-specific light contributions of the two stars are coupled using the predictions from model atmospheres. We found this to provide similar fitted parameters as for solutions in Mode 0 (see Paper I) but a significantly lower $\chi^{2}$. wD2004 unfortunately does not include predictions for the Kepler passband, so we calculated solutions for the Johnson $V$ and $R$ and the Cousins $R$ passbands. We fixed the effective temperature of star A at $21700 \mathrm{~K}$ and fitted for the effective temperature of star B. Whilst our final results 
Table 3. Summary of the parameters for the wD2004 solution of the Kepler SC light curve of V380 Cyg. For further details on the control parameters, please see the WD2004 user guide (Wilson \& Van Hamme 2004). A and B refer to the primary and secondary stars, respectively. The uncertainties on the photometric parameters come from the scatter of appropriate solutions with different sets of fitted parameters, and are much greater than the formal errors of the best single solution.

\begin{tabular}{|c|c|c|}
\hline Parameter & wD2004 name & Value \pm error \\
\hline \multicolumn{3}{|l|}{ Control and fixed parameters: } \\
\hline wD2004 operation mode & MODE & 2 \\
\hline Treatment of reflection & MREF & 2 (detailed) \\
\hline Number of reflections & NREF & 2 \\
\hline Limb darkening law & LD & 1 (linear) \\
\hline Numerical grid size (normal) & $\mathrm{N} 1, \mathrm{~N} 2$ & 60 \\
\hline Numerical grid size (coarse) & $\mathrm{N} 1 \mathrm{~L}, \mathrm{~N} 2 \mathrm{~L}$ & 60 \\
\hline \multicolumn{3}{|l|}{ Fixed parameters: } \\
\hline Orbital period (d) & PERIOD & 12.425719 \\
\hline Primary eclipse time (HJD) & HJD0 & 2441256.544 \\
\hline Mass ratio & $\mathrm{RM}$ & 0.6129 \\
\hline$T_{\text {eff }} \operatorname{star} \mathrm{A}(\mathrm{K})$ & TAVH & 21700 \\
\hline Rotation rates & $\mathrm{F} 1, \mathrm{~F} 2$ & $1.5,2.0$ \\
\hline Gravity darkening & GR1, GR2 & 1.0 \\
\hline Bolometric albedos & ALB1, ALB2 & 1.0 \\
\hline Bolometric LD coeff. A & XBOL1 & 0.645 \\
\hline Bolometric LD coeff. B & XвоL2 & 0.681 \\
\hline Third light & EL3 & 0.0 \\
\hline Passband LD coeff. B & $\mathrm{x} 2$ & 0.224 \\
\hline Orbital eccentricity & E & 0.2224 \\
\hline \multicolumn{3}{|l|}{ Fitted parameters: } \\
\hline Phase shift & PSHIFT & $-0.0502 \pm 0.0003$ \\
\hline Star A potential & PHSV & $4.676 \pm 0.020$ \\
\hline Star B potential & PHSV & $11.06 \pm 0.11$ \\
\hline Orbital inclination $\left({ }^{\circ}\right)$ & XINCL & $80.76 \pm 0.24$ \\
\hline Longitude of periastron $\left({ }^{\circ}\right)$ & PERR0 & $138.4 \pm 0.39$ \\
\hline$T_{\text {eff }}$ star B (K) & TAVC & $23840 \pm 500$ \\
\hline Light from star A & HLUM & $12.045 \pm 0.006$ \\
\hline Passband LD coeff. A & $\mathrm{x} 2$ & $0.137 \pm 0.064$ \\
\hline \multicolumn{3}{|l|}{ Derived parameters: } \\
\hline Light from star B & CLUM & $0.750 \pm 0.012$ \\
\hline Fractional radius of star A & & $0.2633 \pm 0.0015$ \\
\hline Fractional radius of star B & & $0.06403 \pm 0.00072$ \\
\hline \multicolumn{3}{|l|}{ Physical properties: } \\
\hline Mass of star $\mathrm{A}\left(\mathrm{M}_{\odot}\right)$ & & $11.43 \pm 0.19$ \\
\hline Mass of star $\mathrm{B}\left(\mathrm{M}_{\odot}\right)$ & & $7.00 \pm 0.14$ \\
\hline Radius of star $\mathrm{A}\left(\mathrm{R}_{\odot}\right)$ & & $15.71 \pm 0.13$ \\
\hline Radius of star B $\left(\mathrm{R}_{\odot}\right)$ & & $3.819 \pm 0.048$ \\
\hline Surface gravity star A $(\log g)$ & & $3.104 \pm 0.006$ \\
\hline Surface gravity star B $(\log g)$ & & $4.120 \pm 0.011$ \\
\hline Orbital semimajor axis $\left(\mathrm{R}_{\odot}\right)$ & & $59.65 \pm 0.35$ \\
\hline $\log \left(\right.$ Luminosity $\left.\mathrm{A} / \mathrm{L}_{\odot}\right)$ & & $4.691 \pm 0.041$ \\
\hline $\log \left(\right.$ Luminosity $\left.\mathrm{B} / \mathrm{L}_{\odot}\right)$ & & $3.626 \pm 0.038$ \\
\hline$M_{\mathrm{bol}}$ star A (mag) & & $-6.98 \pm 0.10$ \\
\hline$M_{\mathrm{bol}} \mathrm{star} \mathrm{B}(\mathrm{mag})$ & & $-4.31 \pm 0.10$ \\
\hline Distance (pc) & & $970 \pm 21$ \\
\hline
\end{tabular}

yield an uncertainty of roughly $500 \mathrm{~K}$ on the effective temperature of star B, the choice of different passbands moves the best-fitting value around by no more than $60 \mathrm{~K}$. We therefore used the Cousins $R$ passband in our final solutions, and added an extra $\pm 100 \mathrm{~K}$ on to the uncertainties of the effective temperature of star B.

Our exploratory fits revealed that the light curve yields poorer constraints on the orbital shape ( $e$ and $\omega$ ) than expected. The quantities $e$ and $\omega$ are strongly correlated, a common situation when studying eclipsing binaries, making it possible to match the light curve for $0.20<e<0.24$. The time difference between adjacent primary and secondary eclipses puts an excellent constraint on $e \cos \omega$, making this quantity well determined, whereas spectroscopy yields the quantity $e \sin \omega$ to a good precision. Without the ability to fit directly for these quantities in either WD2004 or in SPD, we instead constrained the orbital shape by iterating between the two analyses. We used the light curve to find the best $\omega$ for a given $e$, and the disentangling to find the best $e$ for a given $\omega$. This procedure converged quickly to a solution which well satisfied both types of data, and the final solutions for the light-curve analysis were performed with $e$ fixed at 0.2224 .

For our adopted solution, we fitted for a phase shift with respect to the orbital ephemeris of G2000, the potentials of the two stars, $\omega$, the orbital inclination, the passband-specific light contribution and linear LD coefficient of star A and the effective temperature of star B. We obtained fits for the full (binned) data as well as for the six quarters individually. Our final values for the fitted parameters refer to the best fit to the full data, and our error bars are the standard deviation of the values from the six individual quarters. These results are given in Table 3, and the best fits are plotted in Fig. 2. We also give the fractional radii (stellar radii divided by the orbital semimajor axis) which are needed to calculate the physical properties of the two stars.

It might be justified to divide the error bars of our final parameters by $\sqrt{6}$, as we modelled the six quarters of Kepler data separately, but we have refrained from doing so. This more conservative approach reflects the reality of the situation: the WD2004 code is not capable of fitting the data perfectly (see Fig. 2), and the dominant 'noise' (i.e. signal not arising from the effects of binarity) is due to the intrinsic variability of the primary rather than to Poisson statistics. The final parameters are nevertheless very well determined, and lead to measurements of the masses and radii of the stars to precisions approaching 1 percent. Agreement with the parameters found by previous studies (P2009 and Paper I) is also good. The error bars are typically an order of magnitude larger than the formal errors calculated by WD2004 from the covariance matrix, as expected due to the intrinsic variability of the primary.

Table 3 also contains the full physical properties of the V380 Cyg system, calculated using the ABSDIM code (Southworth, Maxted \& Smalley 2005) and our new spectroscopic and photometric results. For the distance, we quote the value obtained using the 2MASS $K_{s}$ apparent magnitude, bolometric corrections from Bessell, Castelli \& Plez (1998) and adopting a reddening of $E(B-$ $V)=0.21 \pm 0.03 \mathrm{mag}$.

\subsection{Frequency analysis}

The residuals obtained after the orbital fit subtraction were subjected to further frequency analysis, performed on both the combined data set and the data from individual quarters separately. For the extraction of individual frequencies, amplitudes and phases, we used the Lomb-Scargle version of the discrete Fourier transform (DFT; Lomb 1976; Scargle 1982) and consecutive prewhitening. A more detailed mathematical description of the procedure can be found in Degroote et al. (2009).

The highest amplitude $(\sim 1 \mathrm{mmag})$ contribution of $0.08054 \mathrm{~d}^{-1}$ detected in the residuals of all data sets is close to the orbital frequency $f_{\text {orb }}=1 / P=0.08048 \mathrm{~d}^{-1}$. The difference between the two values is insignificant given the Rayleigh limit of $0.0013 \mathrm{~d}^{-1}$ for our photometric data. We also detected a series of higher harmonics of the orbital frequency with amplitudes at least twice as low. 


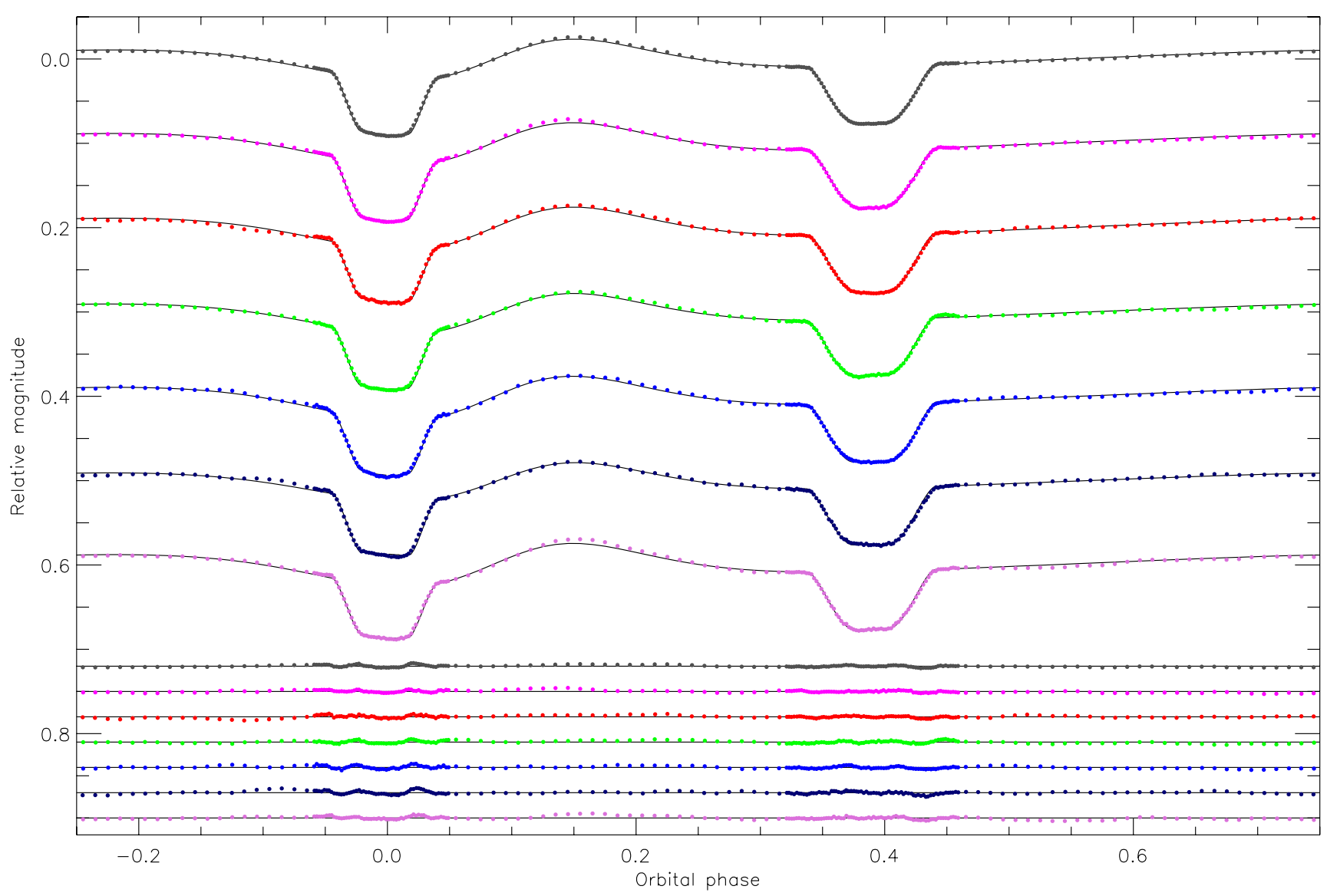

Figure 2. Best fits of the Kepler satellite light curves of V380 Cyg using wD2004. The binned data are shown in the upper part of the plot and the residuals of the fits in the lower part of the plot. The fits are shown for the combined data and for individual quarters.

The second dominant frequency in the combined data set was found to be of $0.12946 \mathrm{~d}^{-1}(1.4978 \mu \mathrm{Hz})$. The analysis performed on the data from individual quarters showed that this peak is variable in time, both in frequency and amplitude. We also detected the second harmonic at $0.25879 \mathrm{~d}^{-1}(2.9942 \mu \mathrm{Hz})$ of this frequency, and together with the orbital frequency, these are the three dominant peaks in the combined data set (left part of Table 8, labelled 'Photometry'). From the determined radius, inclination angle and projected rotational velocity of the primary (cf. Table 3 and Section 4.2), we estimate the true rotation frequency of the star to be $\sim 0.1250 \mathrm{~d}^{-1}(1.4463 \mu \mathrm{Hz})$. This value is slightly different from $0.12946 \mathrm{~d}^{-1}(1.4978 \mu \mathrm{Hz})$ deduced from the observed light variations, which is not unexpected given that the former value represents the rotational frequency averaged over the stellar interior. We thus speculate that the frequency of $0.12946 \mathrm{~d}^{-1}$ is associated with the rotationally modulated signal originating from the primary component of V380 Cyg. The signal itself was found to be slightly non-sinusoidal, and the higher frequency value (compared to the true rotation frequency) might be evidence for differential rotation (in the sense that the spot rotates faster than the bulk of the star). We explore the possibility of observing the spot signal in more detail in Section 4.5 .

In total, we detected about 300 significant frequencies in the combined data set assuming an $\mathrm{S} / \mathrm{N}$ of 4.0 as a stop criterion (Breger et al. 1993). Fig. 3 shows all significant frequencies and their amplitudes extracted from the combined data set; those possibly contaminated by the binary orbit are indicated by the thick red lines. We can see that the frequency density is rather high and that there is a clear

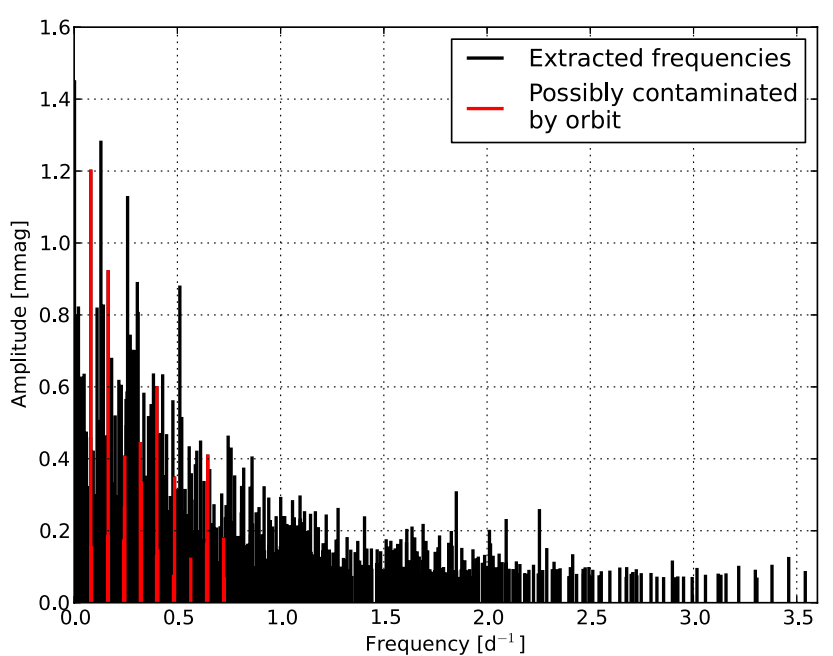

Figure 3. Results of the frequency analysis of the photometric residual signal after subtraction of the orbital solution from the combined data set. Thick red lines indicate the peaks possibly contaminated by the orbit.

trend of decreasing amplitude as the frequency increases. The timefrequency analysis performed on individual quarters showed that the signal is variable both in appearance and amplitude, suggesting its stochastic nature. This confirms our earlier findings presented in Paper I. 


\subsection{Interpretation of the intrinsic variability of the primary}

The strong correlation between amplitude and frequency suggests that the signal arises from a stochastic background signal, rather than oscillations. Stochastically excited oscillations are expected to be excited in a particular region in the frequency spectrum, with a cut-off at the lower end instead of a continuous increase in amplitude. To verify this, we first fitted a granulation signal to the power spectrum of our residual signal (cf. Fig. 4). The original signal is shown in black, whereas its smoothed version is shown as a grey solid line. The fit has been performed to the smoothed curve assuming a granulation signal in combination with the white noise (grey dash-dotted line). The increasing amplitude at lower frequencies is clearly visible, confirming the hypothesis of a stochastic background signal. Secondly, we explore the hypothesis of stochastically excited g modes in more detail. In the case of stochastically excited p modes (i.e. solar-like oscillations), one expects a 'bump' to occur in the power spectrum at the location where the frequencies are excited. To check whether this is applicable to the g-mode spectrum as well, we simulated solar-like stochastic oscillations but with the g-mode frequencies. As expected, a cut-off at low frequencies shows up producing a clear 'bump' in the power spectrum where the frequencies get excited. The results of the simulation are in contradiction with what is observed in V380 Cyg, again implying that the observed low-amplitude signal is dominated by some stochastic 'background noise'.

Blomme et al. (2011) investigated three O-type stars, HD 46223, HD 46150 and HD 46966, based on high-quality light curves gathered with the CoRoT (Auvergne et al. 2009) space mission. The three studied objects are located in the HR diagram just at the boundary of the instability strip due to the $\kappa$-mechanism. However, the amplitude spectra of all three stars were found to be unlike any of known types of pulsating stars. That is, the spectra show a clear trend of increasing amplitude as the frequency decreases; the individual frequencies are found to vary both in appearance and in time, suggesting a stochastic nature of the signal. The authors concluded that most of the power detected in the periodograms of these three stars can be ascribed to 'red noise', which has nothing to do with the instrumental noise but rather points to (predominantly) stochastic behaviour. This red noise is assumed to be intrinsic to the star, and

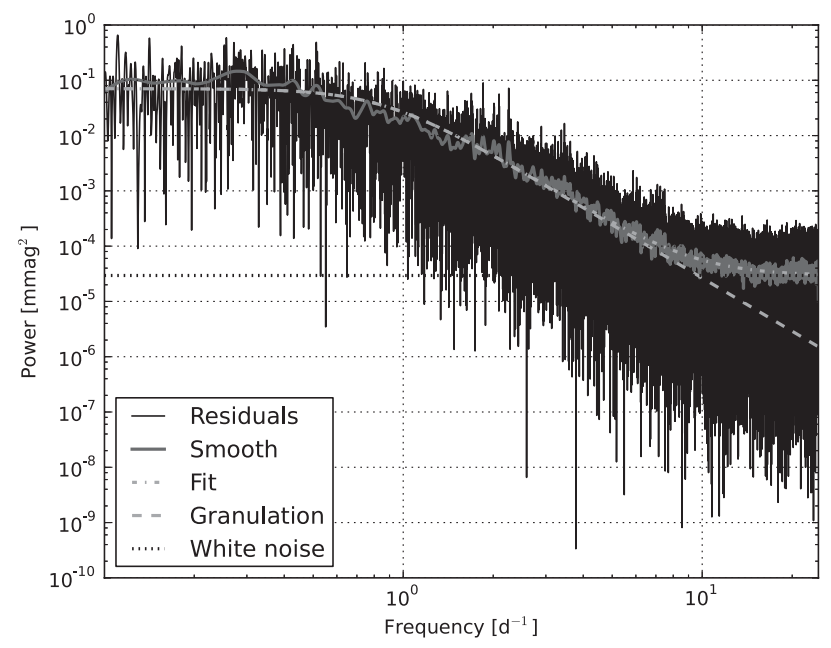

Figure 4. Power spectrum (black) of the orbital residual signal of V380 Cyg with the fit by a granulation (grey dashed line) and a granulation + white noise (grey dash-dotted line) signal. The grey solid line represents a smoothed power signal to which the fit has been performed.
Blomme et al. (2011) discussed three possibilities for its physical cause: sub-surface convection, granulation and inhomogeneities in the stellar wind. These authors stressed that all three options are highly speculative, although previous studies already showed a link between convection and red noise (Schwarzschild 1975), reported about observational evidence of clumping in stellar winds of earlytype stars (Puls, Vink \& Najarro 2008), as well as the existence of a sub-surface convective layer that manifests itself as a large microturbulent velocity field, the generation of magnetic fields and line-profile variations (LPV; Cantiello et al. 2009).

Neiner et al. (2012) presented a study of the CoRoT Be star HD 51452. The authors reported the detection of rather highamplitude (0.5-1.0 mmag) oscillations in the g-mode regime for this B0 star, whereas no (significant) power in the p-mode regime is present. They found that the star has close-to-solar chemical composition and is too hot for $\mathrm{g}$ modes to be excited by the $\kappa$-mechanism. Neiner et al. (2012) proposed a stochastic excitation mechanism in the gravito-inertial regime as the most realistic explanation of the low-frequency oscillations detected in CoRoT photometry of HD 51452, where the modes are assumed to be excited either in the convective core or in a thin convective sub-surface layer. Though the shape of the amplitude spectrum of HD 51452 is different from those reported by Blomme et al. (2011) for three O-type stars in the CoRoT field, both studies concluded that theoretically predicted modes could not be detected in their objects, and that the observed frequencies are too much off from the theoretical ones.

Shiode et al. (2013) investigated the excitation and propagation of stochastic internal gravity waves as well as their detectability in terms of flux and RV variations at the surfaces of massive stars. The authors found that the convective core of high-mass, main-sequence stars might be responsible for the excitation of these modes which cause intrinsic photometric variability at frequencies of $0.4-0.8 \mathrm{~d}^{-1}$ $(5-10 \mu \mathrm{Hz})$ with surface amplitudes of some tens of micromagnitudes. As the stars evolve along the main sequence, oscillations at frequencies down to $0.08 \mathrm{~d}^{-1}(\sim 1 \mu \mathrm{Hz})$ become detectable and the surface flux variability reaches the level of some hundred micromagnitudes. These theoretical predictions are in agreement with the results of Blomme et al. (2011), who detected power at the same frequencies and amplitudes in amplitude spectra of three O-type stars. However, the theory is found to disagree with the observations regarding the mode lifetimes: while Blomme et al. (2011) found mode lifetimes to be of the order of days to hours, the theoretical predictions of Shiode et al. (2013) suggested lifetimes of years to Myrs. Rogers et al. (2013) also found that internal gravity waves can effectively carry angular momentum within a star and might cause spin-up or spin-down of the outer layers of the star and might be responsible for the Be phenomenon observed in some B-type stars.

The signal we observe in the primary component of V380 Cyg is very similar to that observed by Blomme et al. (2011) in their three O-type stars: it is clearly not instrumental, it is variable both in appearance and amplitude on short time-scales (see fig. 3 of Paper I), and it has low amplitude at the level of some hundred micromagnitudes. These characteristics of the observed signal are not in contradiction with the theoretical predictions by Shiode et al. (2013) and Rogers et al. (2013) for stochastically excited gravity waves. We will show that the primary of V380 Cyg exhibits a high microturbulent velocity. Both Cantiello et al. (2009) and Shiode et al. (2013) showed that thin convective sub-surface layers present in some massive stars can be another important source of g modes near the stellar surface. The authors found that these modes are characteristic of degree $l>30$, which implies that they cannot produce large surface 
brightness variations, but still could be responsible for large microturbulent fields observed in massive stars. The high microturbulent velocity observed in the primary of V380 Cyg supports this theory. On the other hand, the large microturbulent velocity field can only be considered as an indirect confirmation of the theory, while such high-degree non-radial modes cannot be directly observed because of their very low amplitudes in the disc integrated light.

\section{SPECTROSCOPIC ANALYSIS}

In this section, we present a detailed spectroscopic analysis of the V380 Cyg system based on the high-quality spectroscopic data described in Section 2. The analysis includes determination of the orbital parameters and the disentangled spectra of both components by means of the SPD technique, estimation of the atmospheric parameters and chemical composition for both stars as well as further comparison with the evolutionary models, and the frequency analysis and the Doppler imaging (DI) analysis of the selected spectral lines in the spectrum of the primary component.

\subsection{Spectral disentangling}

We use the spectral disentangling (SPD) method (Simon \& Sturm 1994) to determine the orbital elements for the V380 Cyg system. The method enables simultaneous separation of the intrinsic individual spectra of the components and determination of the orbital parameters in a self-consistent way from time series of the observed composite spectra of a binary system. In this sense, the measurements of the RVs of the components from usually complex blends of spectral lines are by-passed, and a set of the orbital elements is optimized instead.

SPD has been performed in Fourier space as formulated by Hadrava (1995) and implemented in the FDBINARY code (Ilijic et al. 2004). Such implementation is less demanding on the memory storage, and on the CPU time than the original method introduced by Simon \& Sturm (1994). The DFT is used in the FDBINARY code which enables more freedom in the selection of the spectral regions as well as an arbitrary number of selected pixels which allows better preservation of the original spectral resolution of the input spectra.

Spectral lines of both stars get distorted in the course of the corresponding eclipses due to the Rossiter-McLaughlin effect (McLaughlin 1924; Rossiter 1924). Therefore, the principal condition of SPD stating that no variation other than that due to orbital motion should be present in the spectra is violated at these phases. Hence, only out-of-eclipse spectra of V380 Cyg have been selected by us for the SPD analysis. However, without any significant change in the fractional light contribution of the components in the course of the orbital cycle, the zero-order Fourier mode becomes singular. This leads to an ambiguity in the obtained disentangled spectra - external information on the light ratio is needed for their proper renormalization (cf. Pavlovski \& Hensberge 2005, 2010; Pavlovski \& Southworth 2009). Moreover, undulations in the disentangled spectra might appear but this unwanted disturbance could be handled with appropriate phase coverage of the input spectra (Hensberge, Ilijić \& Torres 2008).

Out of 452 spectra, 237 measurements were taken at the out-ofeclipse phases. The optimization in the FDBINARY code is performed by means of the simplex algorithm. In order to avoid possible local minima in the solution, we ran a very large number of independent runs which makes SPD quite demanding on CPU time even with the reduced (to 237) number of input spectra. From the previous studies (G2000; P2009; Paper I), it is known that the effective temperatures of the components are similar $(\sim 22000 \mathrm{~K})$, and are in the range of the maximum intensity of $\mathrm{He} \mathrm{I}$ lines. This is a fortunate situation for the quality of the determination of the orbital parameters in the case of a large light ratio between the components. Mainly due to their large intrinsic width, Balmer lines are not suitable for the accurate determination of the orbital elements. Thus, the orbital elements were derived by focusing on several wavelength intervals centred at the prominent $\mathrm{He}$ I lines at $\lambda \lambda 4026,4388,4471,4713,4920$ and $6678 \AA$; the mean orbital elements are listed in the last column of Table 4 . The errors quoted are the standard deviations of the mean. These are not realistic (systematic) errors but rather illustrate an excellent consistency between the orbital elements derived from different spectral regions.

A portion of the components' disentangled spectra centred at $\mathrm{H} \gamma$ and including two $\mathrm{He}_{\mathrm{I}}$ and one $\mathrm{C}_{\mathrm{II}}$ lines is illustrated in Fig. 5. The faintness of the secondary (upper spectrum) is obvious despite its similar effective temperature to the primary's. We used the orbital solution from Table 4 (last column) to do the SPD in a wide wavelength range that, besides helium and metal lines, also included three Balmer lines $(\mathrm{H} \delta, \mathrm{H} \gamma$ and $\mathrm{H} \beta$ ). We did not consider the red part of the optical spectrum beyond $580 \mathrm{~nm}$ for the SPD to prevent the influence of the telluric spectrum on the resulting disentangled spectra. Only those few regions the least influenced by the telluric contributions and centred at some isolated and prominent metal (or helium) lines were subjected to SPD in that part of the spectrum (e.g. a couple of strong lines of $\mathrm{C}_{\text {II }}$ at 6578 and $6582 \AA$, He I line at $6678 \AA$ A, etc.).

Table 4 compares our orbital solution with the most recent findings from the literature. A comparison between the different solutions is not straightforward, however, mainly because the corresponding studies were based on spectra of different quality and quantity. The largest deviations are found for the RV semi-amplitude of the secondary component, $K_{\mathrm{B}}$, and thus for the mass ratio, $q$. The main reason for this is the small spectral contribution of the mainsequence companion to the composite spectra of the binary, given its faintness compared to the evolved primary component. Indeed, one of the most recent studies by P2009 involving high-resolution spectroscopy was based on the same method of SPD but provided a significantly higher value of $K_{\mathrm{B}}$ (and thus a lower mass ratio) compared to our findings. We suppose the main reason for this discrepancy is a lower $\mathrm{S} / \mathrm{N}$ of and a poor orbital phase coverage provided by the spectra analysed in P2009. Herewith, with about 240 spectra used for the analysis, we have almost perfect phase coverage, except for the omitted spectra at the phases of both eclipses.

\subsection{Spectrum analysis of both binary components}

As mentioned above, the SPD method, along with the orbital solution, provides disentangled spectra of the individual stellar components of a binary system. The spectra can be analysed by methods suitable for single stars to determine the effective temperatures, surface gravities and chemical composition of the two stars to a high precision (Hensberge, Pavlovski \& Verschueren 2000; Frémat, Lampens \& Hensberge 2005; Pavlovski \& Hensberge 2005; Clausen et al. 2008; Hareter et al. 2008; Pavlovski \& Southworth 2009; Tkachenko, Lehmann \& Mkrtichian 2009). In particular, the effective temperature determined from hydrogen line profiles, and/or strength of the temperature-sensitive lines, superseded in accuracy and precision those derived from e.g. broad-band or Strömgren photometry. The same holds for the metallicity derived from a detailed abundance study of stellar spectra rather than photometric colour indices. 
Table 4. Comparison of the orbital elements of the V380 Cyg system derived by us with the results of previous studies. $T_{0}(2400000+)$ stands for the time of periastron passage, $e$ for the orbital eccentricity, $\omega$ for the longitude of the periastron, $q$ for the mass ratio, and $K_{\mathrm{A}}$ and $K_{\mathrm{B}}$ for the RV semi-amplitude of the star A and B, respectively. Our errors are represented by standard deviations of the mean and thus do not resemble real error bars.

\begin{tabular}{lcccccc}
\hline Parameter & Unit & $\begin{array}{c}\text { Hill \& Batten } \\
(1984)\end{array}$ & $\begin{array}{c}\text { Lyubimkov } \\
\text { et al. (1996) }\end{array}$ & $\begin{array}{c}\text { Guinan } \\
\text { et al. (2000) }\end{array}$ & $\begin{array}{c}\text { Pavlovski } \\
\text { et al. (2009) }\end{array}$ & $\begin{array}{c}\text { Present } \\
\text { work }\end{array}$ \\
\hline$T_{0}$ & JD & $37455.10 \pm 0.11$ & $37454.974 \pm 0.054$ & $49495.70 \pm 0.09$ & $54615.18 \pm 0.14$ & $54602.888 \pm 0.007$ \\
$e$ & & $0.22 \pm 0.01$ & $0.2183 \pm 0.0051$ & 0.22 & $0.206 \pm 0.008$ & $0.2261 \pm 0.0004$ \\
$\omega$ & degree & $127.6 \pm 2.8$ & $122.2 \pm 1.8$ & $132.7 \pm 0.3$ & $134.2 \pm 1.1$ & $141.5 \pm 0.2$ \\
$K_{\mathrm{A}}$ & $\mathrm{km} \mathrm{s}^{-1}$ & $92.8 \pm 2.6$ & 93.95 & $95.6 \pm 0.5$ & $95.1 \pm 0.3$ & $93.54 \pm 0.07$ \\
$K_{\mathrm{B}}$ & $\mathrm{km} \mathrm{s}^{-1}$ & $160.7 \pm 2.8$ & 155.3 & $147.9 \pm 1.2$ & $160.5 \pm 1.2$ & $152.71 \pm 0.22$ \\
$q$ & & $0.577 \pm 0.019$ & 0.605 & $0.646 \pm 0.004$ & $0.592 \pm 0.005$ & $0.613 \pm 0.001$ \\
\hline
\end{tabular}

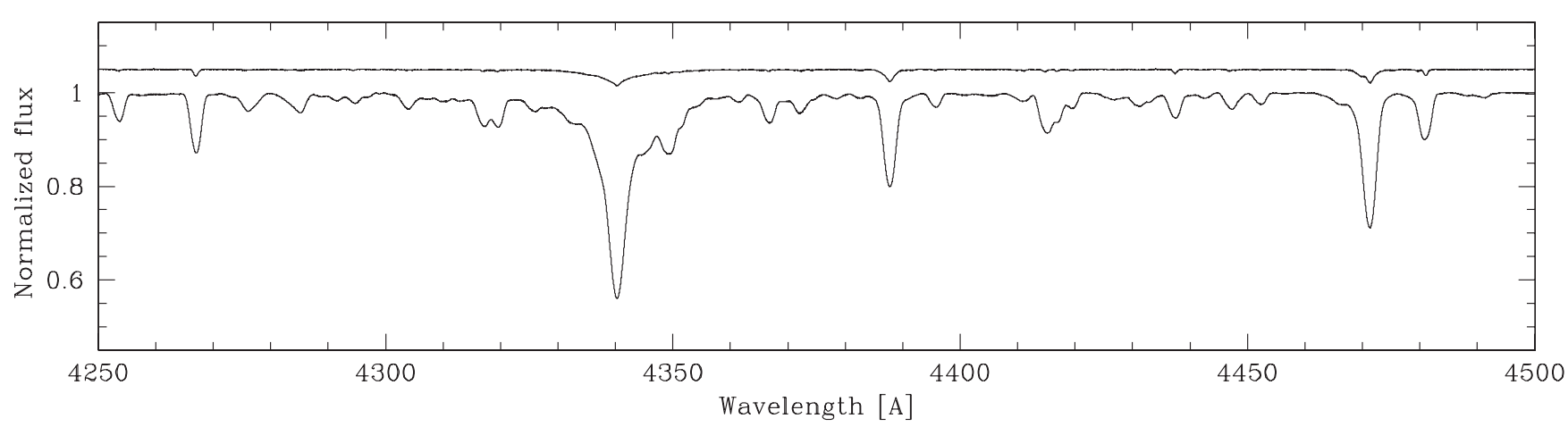

Figure 5. Disentangled spectra of both components of the binary system V380 Cyg in the region of H $\gamma, \mathrm{C}_{\text {II }} 4267 \AA$, and He I 4388 and $4471 \AA$ spectral lines. The spectrum of the secondary was shifted up in the vertical direction for clarity.

Determination of the effective temperature from hydrogen lines of the Balmer series in OB stars suffers from degeneracy in $T_{\text {eff }}$ and $\log g$. This degeneracy is a major limitation in setting up of the appropriate model atmosphere for a detailed abundance study. Thankfully, complementary analysis of the light and RV curves (variations) in detached eclipsing binaries enables the determination of the components' masses and radii with high accuracy. Knowledge of these two fundamental characteristics of the star ensures that the surface gravity is also known to a high precision ( 0.01 dex or better). Hence, the above-mentioned degeneracy in $T_{\text {eff }}$ and $\log g$ can be safely removed for this type of stars.

In the present analysis, we renormalize the disentangled spectra using the light ratio $\left(l_{\mathrm{B}} / l_{\mathrm{A}}=0.062 \pm 0.001\right)$ derived in the course of the light-curve analysis (cf. Section 3.1). The effective temperatures of the components are then determined by means of the optimal fitting of the renormalized spectra of the individual stellar components to a grid of theoretical spectra. Since we are dealing with B2 spectral type stars, the synthetic spectra were computed assuming non-LTE line formation (where 'LTE' stands for the local thermodynamical equilibrium). However, the whole analysis is based on the so-called hybrid approach that assumes LTE-based atmosphere models computed with the ATLAS9 code (Kurucz 1993) and non-LTE spectral synthesis with detailed statistical balance by means of the DETAIL (Butler 1984) and SURFACE (Giddings 1981) codes. Justification of such an approach has been discussed in detail in Nieva \& Przybilla (2007). The grid of theoretical spectra ranges from 18000 to $25000 \mathrm{~K}$ with a step width of $1000 \mathrm{~K}$ in effective temperature and from 3.0 to 4.5 dex with a step size of 0.1 dex in surface gravity. Details on input atomic data are given in Nieva \& Przybilla (2012).

The optimal fitting was performed with the genetic algorithm as implemented in the PIKAIA subroutine after Charbonneau (1995). Besides the effective temperature for each binary component, we also optimized for the projected rotational velocities, the velocity offset between the disentangled spectra and the rest-frame wavelengths of the theoretical spectra, and the continuum correction (vertical shift in flux). In practice, echelle blaze orders that contain broad Balmer lines are difficult to normalize and merge. In turn, the corresponding spectral regions are the main source of difficulties in the SPD. In particular, in Fourier-based disentangling, wrong continuum placement usually shows up as undulations in the resulting disentangled spectra (Hensberge et al. 2008). Moreover, due to the fact that we assume the light ratio between the two components to be time independent, the disentangled spectra are not properly normalized and an additive correction value should be applied to return them to the level of unity.

Table 5 lists the derived atmospheric parameters along with some fundamental quantities derived from the light-curve fitting (cf. Section 3.1). The parameters derived spectroscopically are marked with

Table 5. Atmospheric parameters of both components of V380 Cyg along with some fundamental characteristics derived from the light-curve fitting. $V_{\text {eq }}$ and $V_{\text {synch }}$ are the observed equatorial and calculated synchronous rotational velocities, respectively. Error bars are at the $1 \sigma$ level, with $\sigma$ the standard deviation of the mean.

\begin{tabular}{llcc}
\hline Parameter & Unit & Star A & Star B \\
\hline $\log g^{a}$ & $\mathrm{~cm} \mathrm{~s}^{-2}$ & $3.104 \pm 0.006$ & $4.120 \pm 0.011$ \\
$T_{\text {eff }}$ & $\mathrm{K}$ & $21700 \pm 300$ & $22700 \pm 1200$ \\
$v_{\text {turb }}$ & $\mathrm{km} \mathrm{s}^{-1}$ & $15 \pm 1$ & $1 \pm 1$ \\
$v \sin i$ & $\mathrm{~km} \mathrm{~s}^{-1}$ & $98 \pm 2$ & $38 \pm 2$ \\
$V_{\text {eq }}$ & $\mathrm{km} \mathrm{s}^{-1}$ & $99.3 \pm 1.0$ & $38.5 \pm 3.0$ \\
$V_{\text {synch }}$ & $\mathrm{km} \mathrm{s}^{-1}$ & $63.7 \pm 0.5$ & $15.6 \pm 0.3$ \\
\hline
\end{tabular}

${ }^{a}$ From the light-curve solution. 

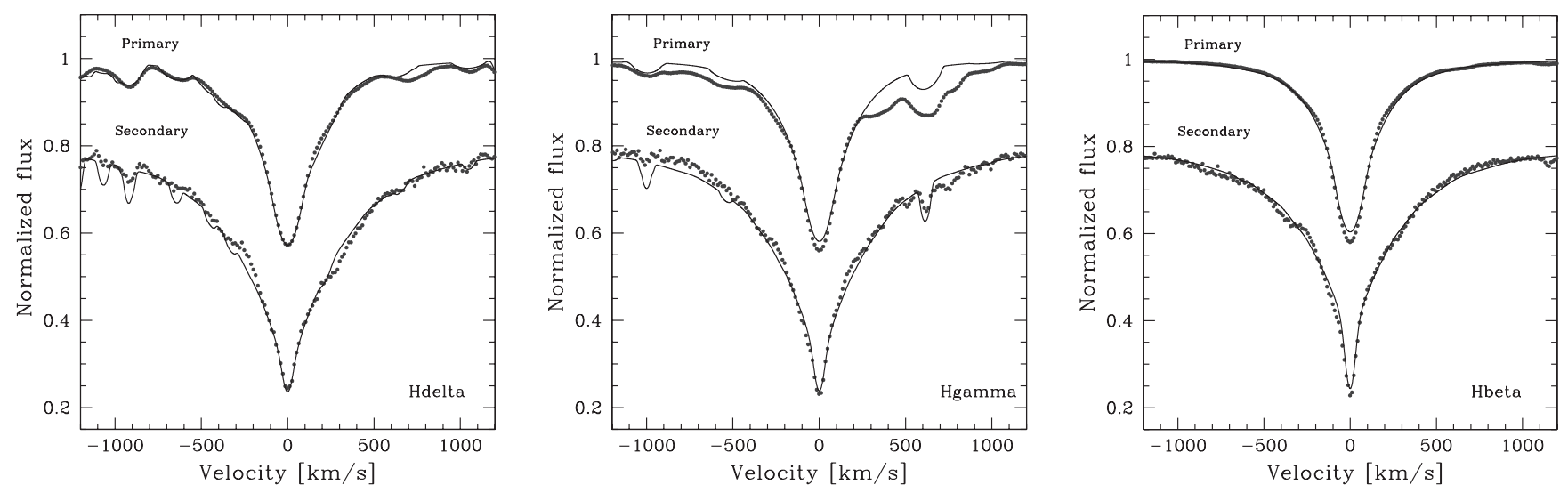

Figure 6. The best-fitting synthetic spectra (lines) in comparison to the renormalized disentangled spectra (dots) of the primary (upper) and the secondary component (lower). From the left- to right-hand panel $\mathrm{H} \delta, \mathrm{H} \gamma$ and $\mathrm{H} \beta$ profiles are shown.

an asterisk ('*'). Effective temperatures were determined from fitting the Balmer lines whereas $v \sin i$ and $v_{\text {turb }}$ were estimated from $\mathrm{He} \mathrm{I}$ and metal lines. Similar to the uncertainties on the orbital elements (cf. Section 4.1), the quoted errors are the standard deviations of the mean. As such, small error bars for the effective temperature of the primary in Table 5 stand for an excellent consistency between the values derived from different Balmer lines. Fig. 6 compares the best-fitting synthetic spectra for both components of V380 Cyg (upper and lower profiles for the primary and secondary, respectively) with the corresponding disentangled spectra, renormalized to the individual continua, in the regions centred at three Balmer lines: $\mathrm{H} \delta$ (left), $\mathrm{H} \gamma$ (middle) and $\mathrm{H} \beta$ (right). The difference in $\mathrm{S} / \mathrm{N}$ between the renormalized spectra of the components is obvious and is due to small contribution of the secondary to the total light of the binary system. The derived effective temperature of the primary compares well to the values reported by G2000 and P2009 (cf. Table 1). Moreover, both authors report about the

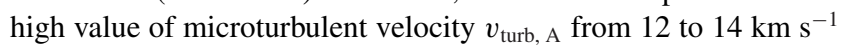
(G2000 and P2009, respectively), though disagree on the metallicity value: $-0.44 \pm 0.07$ dex derived by G2000 to be compared to $-0.02 \pm 0.05$ dex found by P2009. We confirm the high value of the microturbulent velocity: $v_{\text {turb, A }}=15 \pm 1 \mathrm{~km} \mathrm{~s}^{-1}$ from our spectra. Our abundance analysis suggests a similar chemical composition for both binary components (see below); the magnesium abundance of the primary is consistent with the metallicity index reported by P2009.

For the secondary component of V380 Cyg, our spectroscopic and photometric data suggest significantly different values for the effective temperature. While the optimal fitting of the disentangled spectrum of the star results in $T_{\text {eff, B }}=21800 \pm 400 \mathrm{~K}$, a value only slightly above the one found for the primary component, the light-curve modelling delivers the value of $T_{\text {eff, }}$ B $=23840 \pm 500 \mathrm{~K}$ (assuming fixed to the spectroscopic value temperature of the primary, cf. Table 5). Since the disentangled spectra are renormalized using the photometric value of the light ratio between the two stars, we are somewhat in a 'dead cycle': the high photometric effective temperature is inconsistent with the spectral characteristics of the star. As will be shown in Section 4.5, adopting higher effective temperature for the secondary leads to large discrepancy of $\sim 2 \mathrm{M}_{\odot}$ between the mass deduced from binary dynamics and the one obtained from evolutionary models. As a compromise between the two temperatures, we finally adopt the effective temperature of the secondary to be $T_{\text {eff, B }}=22700 \pm 1200 \mathrm{~K}$, i.e. the mean of the spectroscopic and photometric values. Besides $T_{\text {eff }}$ and $v \sin i$,
Table 6. The photospheric helium abundance for both components of the V380 Cyg system as derived from different He I spectral lines.

\begin{tabular}{lccr}
\hline Line & Primary & Secondary & \multicolumn{1}{c}{$\Delta \epsilon$} \\
\hline 4026.2 & $0.087 \pm 0.004$ & $0.096 \pm 0.005$ & $-0.009 \pm 0.006$ \\
4387.9 & $0.062 \pm 0.004$ & $0.105 \pm 0.004$ & $-0.043 \pm 0.006$ \\
4437.6 & $0.085 \pm 0.003$ & $0.081 \pm 0.004$ & $0.004 \pm 0.005$ \\
4471.5 & $0.094 \pm 0.006$ & $0.093 \pm 0.004$ & $0.001 \pm 0.007$ \\
4713.2 & $0.061 \pm 0.003$ & $0.070 \pm 0.004$ & $-0.009 \pm 0.005$ \\
4921.9 & $0.072 \pm 0.004$ & $0.089 \pm 0.004$ & $-0.017 \pm 0.006$ \\
5015.7 & $0.079 \pm 0.005$ & $0.098 \pm 0.005$ & $-0.019 \pm 0.007$ \\
5054.7 & $0.077 \pm 0.005$ & $0.085 \pm 0.005$ & $-0.008 \pm 0.007$ \\
5876.7 & $0.149 \pm 0.013$ & $0.096 \pm 0.006$ & $0.053 \pm 0.014$ \\
6678.1 & $0.083 \pm 0.005$ & $0.108 \pm 0.005$ & $-0.025 \pm 0.007$ \\
Mean & $0.085 \pm 0.019$ & $0.092 \pm 0.015$ & $-0.007 \pm 0.024$ \\
\hline
\end{tabular}

we also derived the microturbulent velocity for the secondary to be $v_{\text {turb, B }}=1 \pm 1 \mathrm{~km} \mathrm{~s}^{-1}$. This parameter is determined for the secondary for the first time; the low value is not unexpected for a B-type star at early phases of the evolution on the main sequence (Lefever et al. 2010; Nieva \& Przybilla 2012; Lyubimkov et al. 2013).

Table 6 lists helium abundances for both stellar components of V380 Cyg. The abundances were measured from 10 individual He I lines in the corresponding disentangled spectra. Within the quoted $(1 \sigma)$ errors, helium abundances of the two binary components agree with each other as well as with the value derived by Nieva \& Przybilla (2012) from a sample of OB stars, but are slightly higher than the solar helium abundance found by Asplund et al. (2009). The quality of the fits for a few selected helium lines in the spectra of both components is shown in Fig. 7. Our results agree with the findings by P2009 in the sense that line-to-line scatter in helium abundance is higher than anticipated. On the other hand, these authors report slightly higher mean helium abundance of $0.094 \pm 0.031$ dex for the primary component derived from the same set of spectral lines, with the two He I lines at 5875.7 and $6678.1 \AA$ showing the highest abundances. A small difference between our and P2009 values can be attributed to slightly different microturbulent velocities derived for the primary component as well as to the difference in the light ratio that was used to renormalize the disentangled spectrum of the star.

Helium abundances of both binary components were also determined by Lyubimkov et al. (1996) based on the equivalent widths 

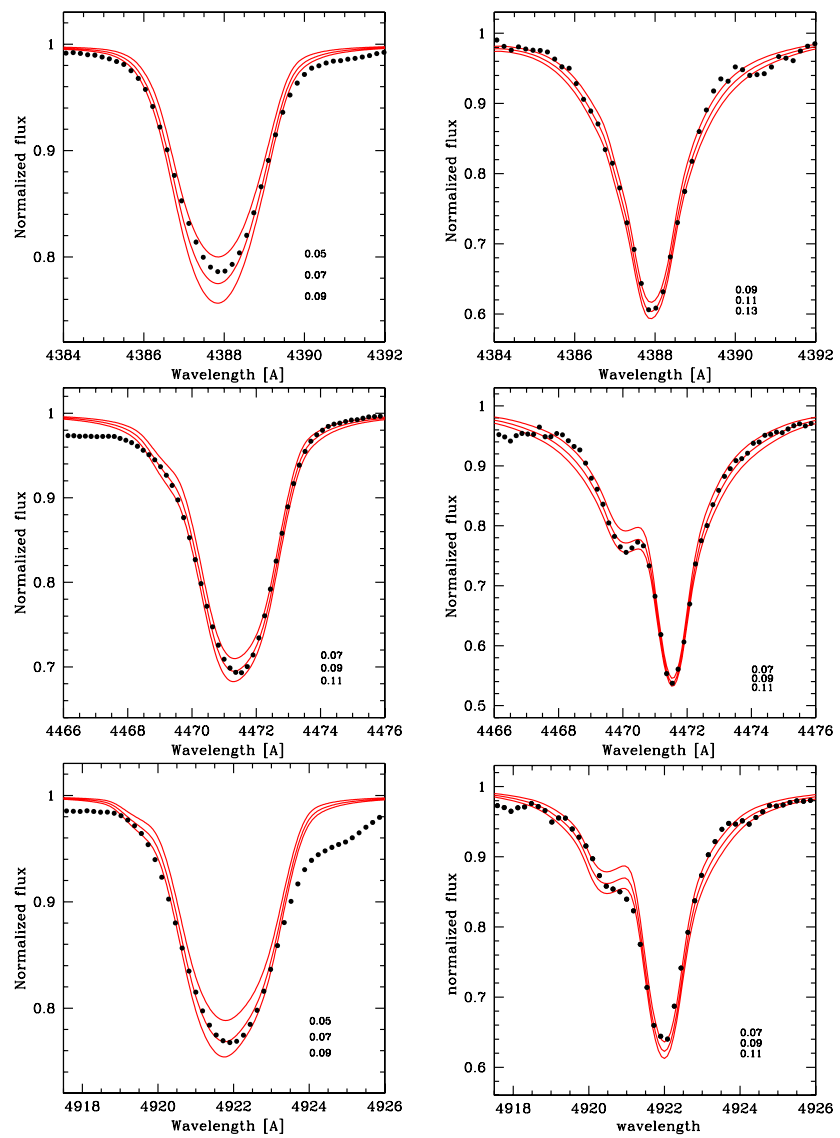

Figure 7. Theoretical spectra (lines) of $\mathrm{He}$ I lines at 4387.9, 4471.5 and $4921.9 \AA$ (from top to bottom) compared to the renormalized disentangled spectra (dots) of the primary (left-hand panels) and the secondary components (right-hand panels). Theoretical spectra are calculated for the stellar parameters listed in Table 5; the assumed different helium abundances are indicated in the bottom-right corner of each panel.

measured directly from the composite spectra of the binary. Given a small contribution of the secondary component of $\sim 6$ per cent to the total light, this way of measuring abundances for this star is associated with large uncertainties. The authors reported a strong helium overabundance of $\sim 0.16 \pm 0.04$ dex for the primary component, with rather large line-to-line scatter. For the secondary, they reported a value of $0.074 \pm 0.009 \mathrm{dex}$ which is lower than both the solar value found by Asplund et al. (2009) and the one derived for a sample of OB stars by Nieva \& Przybilla (2012). Lyubimkov et al. (1996) interpreted their distinctly high helium abundance for the primary component as evidence for a strong internal mixing by turbulent diffusion, which brings $\mathrm{CNO}$ processed material from the core to the surface already during the mainsequence phase. The helium abundances of the binary components derived in our study are in contradiction with the findings and conclusions by Lyubimkov et al. (1996). Similar to the components of V380 Cyg, no excess helium abundance was found in several other high-mass stars, members of binary systems (e.g. Pavlovski \& Hensberge 2005; Pavlovski \& Southworth 2009; P2009; Mayer, Harmanec \& Pavlovski 2013).

Abundances of other species present in the spectra of both components of the V380 Cyg system are given in Table 7. The last column lists the difference between the abundances of the two components. No statistically significant deviations are found for any of the elements, with oxygen and silicon showing the largest discrepancies
Table 7. Same as Table 6 but for metals. Abundances are expressed relative to $\log N(\mathrm{H})=12.0 ; N$ gives the number of used lines.

\begin{tabular}{lrrrrr}
\hline Ion & Component A & $N$ & Component B & $N$ & \multicolumn{1}{c}{$\Delta \epsilon$} \\
\hline $\mathrm{He}$ & $10.97 \pm 0.05$ & 10 & $11.00 \pm 0.03$ & 10 & $-0.03 \pm 0.06$ \\
$\mathrm{C}$ & $8.20 \pm 0.02$ & 6 & $8.19 \pm 0.04$ & 7 & $0.01 \pm 0.04$ \\
$\mathrm{~N}$ & $7.55 \pm 0.05$ & 17 & $7.53 \pm 0.08$ & 19 & $0.02 \pm 0.09$ \\
$\mathrm{O}$ & $8.64 \pm 0.07$ & 20 & $8.55 \pm 0.12$ & 24 & $0.09 \pm 0.14$ \\
$\mathrm{Mg}$ & $7.58 \pm 0.10$ & 3 & $7.63 \pm 0.11$ & 4 & $-0.05 \pm 0.15$ \\
$\mathrm{Si}$ & $7.45 \pm 0.13$ & 7 & $7.29 \pm 0.15$ & 9 & $0.16 \pm 0.20$ \\
$\mathrm{Al}$ & $6.02 \pm 0.11$ & 3 & $5.98 \pm 0.12$ & 3 & $0.04 \pm 0.16$ \\
$\mathrm{~N} / \mathrm{C}$ & $-0.65 \pm 0.05$ & & $-0.66 \pm 0.09$ & & $0.01 \pm 0.10$ \\
$\mathrm{~N} / \mathrm{O}$ & $-1.09 \pm 0.09$ & & $-1.02 \pm 0.15$ & & $-0.07 \pm 0.16$ \\
\hline
\end{tabular}

( $\Delta \log \epsilon=0.09 \pm 0.14$ dex and $0.16 \pm 0.20$ dex, respectively). Thus, we conclude that the two stars have a similar chemical composition or that the difference is smaller than the quoted error bars. Fig. 8 shows fits to some individual carbon and nitrogen lines found in the spectra of both binary components. The synthetic spectra are those used previously for the determination of the fundamental parameters (see above) but computed for different abundances of $\mathrm{C}$ and $\mathrm{N}: \log \epsilon(\mathrm{C})=8.0-8.3 \mathrm{dex}$ and $\log \epsilon(\mathrm{N})=7.4-7.6 \mathrm{dex}$. The dashed line represents the cosmic abundances derived by Nieva $\&$ Przybilla (2012), which were recently found by Lyubimkov et al. (2013) to be a good representation of the $\mathrm{C}$ and $\mathrm{N}$ abundances in some 20 local ( $d \leq 600$ pc from the Sun) B-type main-sequence stars. Obviously, the derived abundances for both components disagree with those reported by Nieva \& Przybilla (2012) and Lyubimkov et al. (2013). However, a large spread in CNO abundances for OB stars was measured (see an overview in Morel 2009). Our abundances agree very well with the average composition of Btype stars found in the compilation of available data by this author. Finally, the derived abundances agree very well with those reported by P2009 for the primary component of V380 Cyg, as well as with the abundances found for other spectral type B members of binary systems: V578 Mon (Pavlovski \& Hensberge 2005) and V453 Cyg (Pavlovski \& Southworth 2009).

\subsection{Frequency analysis of residual spectra}

The individual RV shifts as well as the mean disentangled spectrum of the secondary delivered by the SPD were used to extract the lines of the primary from the original composite spectra. First, the contribution of the secondary has been subtracted from the composite spectra and the residual profiles were then shifted according to the individual shifts to correct for the orbital motion. Moreover, in order to prevent the secondary from influencing our results, similar to the SPD analysis, we additionally excluded all eclipse spectra as they may suffer from the Rossiter-McLaughlin effect (McLaughlin 1924; Rossiter 1924) which serves as an additional 'noise source' for the frequency analysis. The final set of 237 spectra was used to compute RVs as the first-order moment of the line profile as defined by Aerts, de Pauw \& Waelkens (1992), which together with the profiles themselves were the subject of the frequency analysis.

Fig. 9 illustrates time series of $\mathrm{Si}$ III line profiles collected during two single nights (top panel) as well as nine spectra that cover a much longer period of time of about two months (bottom). One can see that the spectra from individual nights do not show any remarkable variation whereas a clear variability is present on a longer time-scale. 

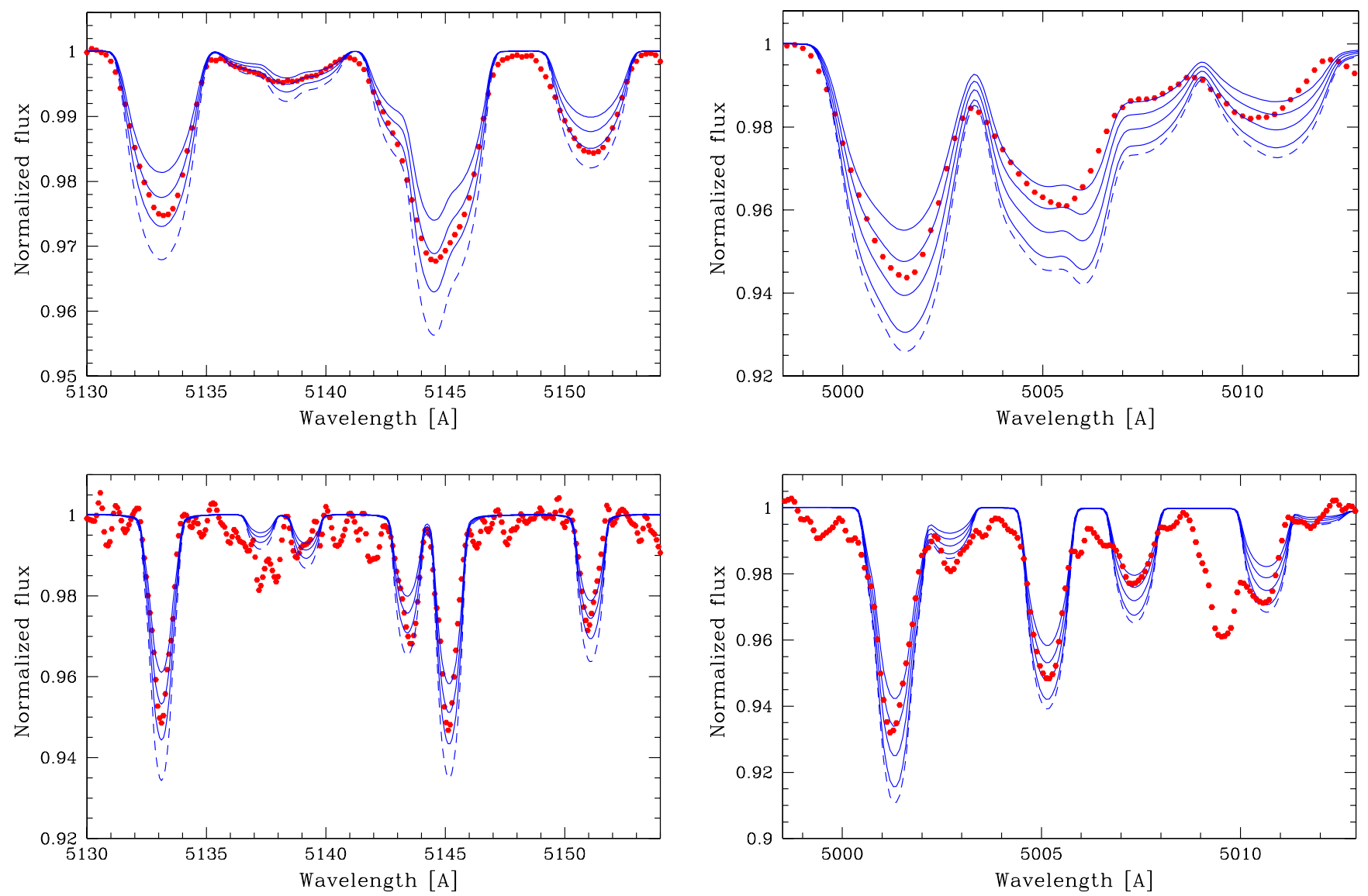

Figure 8. Comparison between the disentangled spectra (symbols) of the primary (top) and secondary (bottom) components with the synthetic spectra (lines) computed assuming different abundances of $\mathrm{C}$ and $\mathrm{N}$. The left- and right-hand panels show bunches of $\mathrm{C}_{\mathrm{I}}$ and $\mathrm{N}_{\mathrm{I}}$ spectral lines, respectively. The carbon $(\log \epsilon(\mathrm{C}))$ and nitrogen $(\log \epsilon(\mathrm{N}))$ abundances range from 8.0 to $8.3 \mathrm{dex}$, and from 7.4 to 7.6 dex, respectively. The step width is equal to 0.1 dex in both cases. Dashed lines correspond to the 'present-day cosmic abundances' as reported by Nieva \& Przybilla (2012).

For the extraction of the individual frequencies from both RVs and line profiles, we used the DFT and the consecutive prewhitening procedure as implemented in the FAMIAS package (Zima 2008). The DFT was computed up to the Nyquist frequency of the data set, and similar to the results of the photometric analysis, no significant contribution was found in the high-frequency domain. At each step of the prewhitening, we optimized the amplitudes and phases of the individual peaks whereas the frequency values were kept fixed. To make sure that no significant contribution was missed in the course of our analysis, we continued extracting frequencies until an $\mathrm{S} / \mathrm{N}$ of 3.0 was reached. However, following the definition of the significance level proposed by Breger et al. (1993) for groundbased data, we consider the frequency to be significant if it has $\mathrm{S} / \mathrm{N} \geq 4$.

In total, we analysed six individual spectral lines: three silicon lines, two lines of helium and one magnesium line. The results of the frequency analysis are summarized in the right part of Table 8, labelled 'Spectroscopy'. The significant frequencies are highlighted in boldface, and each frequency is linked to the photometric one which in turn is indicated in parentheses. Before we start discussing the individual frequencies in more detail, it is worth noting that different lines of the same chemical element delivered compatible results with respect to the significant frequencies. Since our further analysis concerns only these frequencies, in Table 8 , for silicon and helium, we present the results obtained, respectively, from the lines at $\lambda \lambda 4452$ and $4026 \AA$ only.
Obviously, both pixel-by-pixel and moment methods provide consistent results for the lines of all the three chemical elements, in the sense that the signal is detected at the orbital frequency and its low-order harmonics. Indeed, the orbital signal is the only significant one in the first moment of all the analysed lines as well as in the magnesium and helium line profiles. Besides the orbital frequency and its first two harmonics, the pixel-by-pixel based analysis of the silicon line profiles reveals a long-term variability with a frequency of $0.0114 \mathrm{~d}^{-1}(0.1319 \mu \mathrm{Hz})$. A similar frequency of $0.0107 \mathrm{~d}^{-1}(0.1238 \mu \mathrm{Hz})$ was also detected in the photometric data but was found to have very low amplitude $\left(f_{36}\right.$, first column of Table 8). Multiplied by a factor of 11 , this frequency gives a value of $0.1254 \mathrm{~d}^{-1}(1.4509 \mu \mathrm{Hz})$ which is very close to the second, not related to the binary orbit, significant frequency of $0.1261 \mathrm{~d}^{-1}$ $(1.4590 \mu \mathrm{Hz})$ detected in the silicon line profiles. Moreover, the latter frequency in turn agrees within the measurement errors with the rotation frequency of the primary, which we estimated in Section 3.2 to be $\sim 0.1250 \mathrm{~d}^{-1}(1.4463 \mu \mathrm{Hz})$. We thus interpret this signal as being due to the stellar rotation, and suggest that the signal is modulated with a period of about $90 \mathrm{~d}$ (corresponding to the frequency of $0.0114 \mathrm{~d}^{-1}$ or $0.1319 \mu \mathrm{Hz}$ ) which might be evidence for differential rotation. The fact that the rotation signal was detected in silicon lines only suggests an inhomogeneous distribution of the abundance of this element over the stellar surface. In the next section, we verify this hypothesis by applying the DI method to our spectroscopic data. 

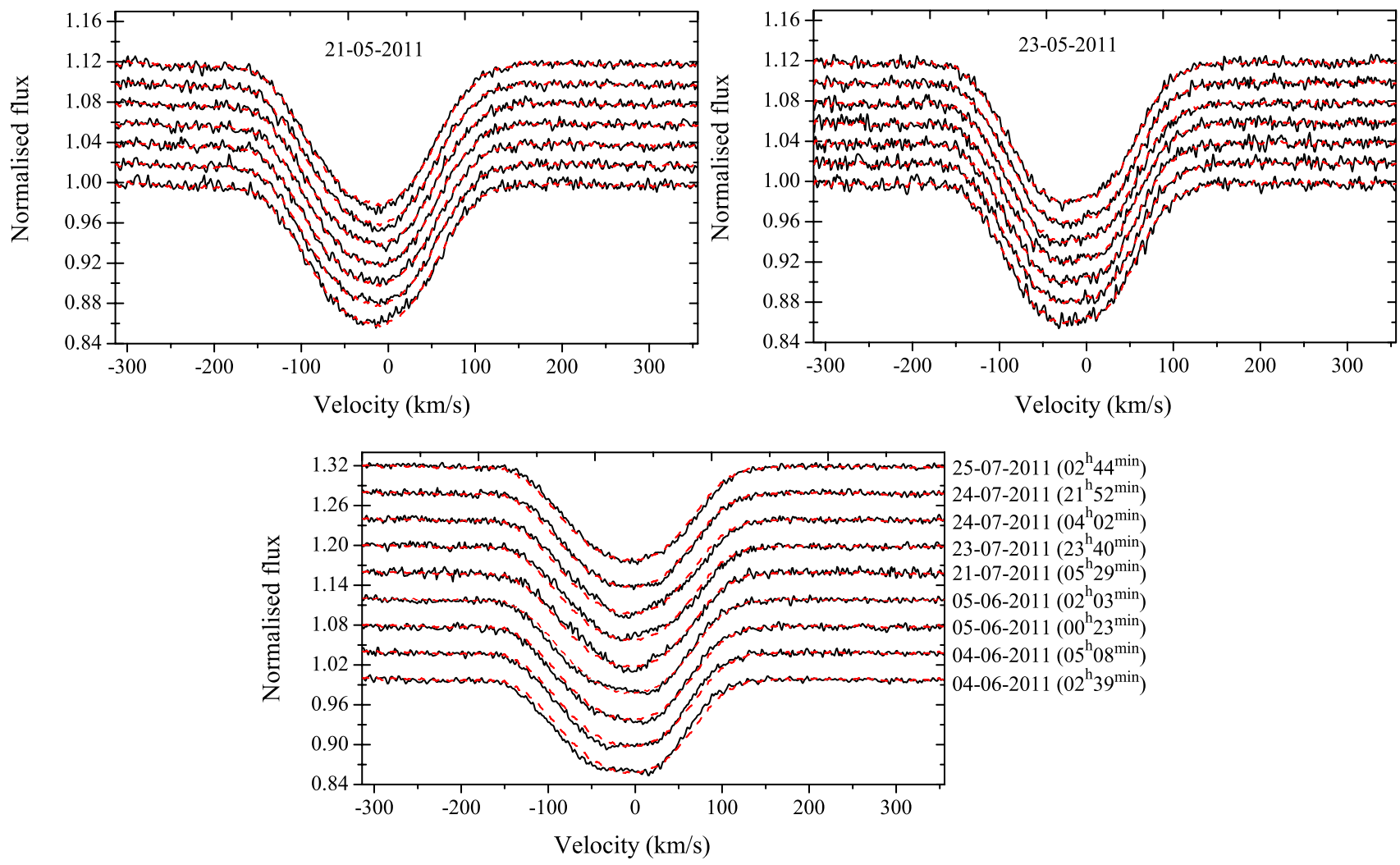

Figure 9. LPV for the primary of V380 Cyg after removing the secondary component and correcting for the orbital motion. Top: time series of spectroscopic observations during a single night (indicated in the plot). Bottom: nine selected spectra covering about a two months period from 2011 June to July. Mean spectrum (red dashed line) is given for comparison for better visibility of the LPV. The value of $v \sin i$ of the star equals $98 \pm 2 \mathrm{~km} \mathrm{~s}^{-1}$ (cf. Section 4.2 .)

Table 8. Results of the frequency analysis. The Rayleigh limit amounts to $0.0013 \mathrm{~d}^{-1}$ for the photometry and $0.0071 \mathrm{~d}^{-1}$ for the spectroscopy. Errors in the amplitude are given in parentheses in terms of last digits. Frequencies matching the significance criterion of S/N $\geq 4.0$ are highlighted in boldface. Each spectroscopic frequency is linked to the photometric one. The corresponding photometric frequency is indicated in parentheses following the value of the spectroscopically derived frequency.

\begin{tabular}{|c|c|c|c|c|c|c|c|c|c|c|c|c|}
\hline & & hotometry & & & & & & Spec & oscopy & & & \\
\hline & & magnitude) & & & & $\begin{array}{l}\text { Pixel-by-pixe } \\
\text { ontinuum uni }\end{array}$ & & & & $\begin{array}{c}\mathrm{RV} \\
\left(\mathrm{km} \mathrm{s}^{-1}\right)\end{array}$ & & \\
\hline$f_{i}$ & Freq $\left(d^{-1}\right)$ & Amplitude & $\mathrm{S} / \mathrm{N}$ & $n \cdot f_{\text {orb }}$ & Freq $\left(d^{-1}\right)$ & Amplitude & $\mathrm{S} / \mathrm{N}$ & $n \cdot f_{\text {orb }}$ & Freq $\left(d^{-1}\right)$ & Amplitude & $\mathrm{S} / \mathrm{N}$ & $n \cdot f_{\text {orb }}$ \\
\hline$f_{1}$ & 0.08054 & $0.001204(215)$ & 5.0 & 1.00 & & & on line & $02 \lambda 455000$ & $.6,4567.8,457$ & $8 \AA$ ) & & \\
\hline$f_{2}$ & $0.12946\left(f_{\text {rot }}\right)$ & $0.001284(208)$ & 5.1 & 1.61 & $0.0114\left(f_{36}\right)$ & $0.0401(26)$ & 4.1 & 0.14 & $\mathbf{0 . 0 8 2 2}\left(f_{1}\right)$ & $0.9900(352)$ & 5.3 & 1.02 \\
\hline$f_{3}$ & $0.25879\left(2 f_{\text {rot }}\right)$ & $0.001131(192)$ & 5.1 & 3.21 & $0.0822\left(f_{1}\right)$ & $0.0090(20)$ & 5.6 & 1.02 & $0.2425\left(f_{52}\right)$ & $0.7767(372)$ & 3.6 & 3.01 \\
\hline$f_{7}$ & 0.14161 & $0.000829(186)$ & 3.6 & & $\mathbf{0 . 2 4 2 6}\left(f_{52}\right)$ & $0.0078(21)$ & 4.1 & 3.01 & $\mathbf{0 . 1 6 3 0}\left(f_{18}\right)$ & $0.6678(393)$ & 4.7 & 2.02 \\
\hline$f_{17}$ & 0.22832 & $0.000606(161)$ & 3.1 & & $0.1606\left(f_{18}\right)$ & $0.0075(22)$ & 4.0 & 1.99 & 2.1014 & $0.3511(358)$ & 3.7 & \\
\hline$f_{18}$ & 0.16474 & $0.000624(159)$ & 3.0 & & $0.6397\left(f_{50}\right)$ & $0.0061(20)$ & 3.4 & & $0.8916\left(f_{99}\right)$ & $0.3853(382)$ & 3.8 & \\
\hline$f_{25}$ & 0.29562 & $0.000589(148)$ & 2.9 & & $\mathbf{0 . 1 2 6 1}\left(f_{2}\right)$ & $0.0063(21)$ & 4.0 & 1.57 & $0.6058\left(f_{165}\right)$ & $0.2268(352)$ & 3.5 & \\
\hline$f_{35}$ & 0.44763 & $0.000469(132)$ & 2.8 & & $0.2909\left(f_{25}\right)$ & $0.0058(21)$ & 3.1 & & & & & \\
\hline$f_{36}$ & 0.01067 & $0.000664(132)$ & 2.8 & & $0.2345\left(f_{17}\right)$ & $0.0052(20)$ & 3.0 & & & & & \\
\hline$f_{50}$ & 0.64702 & $0.000412(117)$ & 2.8 & & & & Telium & nos & $1026.6,4471.5$ & & & \\
\hline$f_{51}$ & 0.48624 & $0.000351(115)$ & 2.8 & & $0.0792\left(f_{1}\right)$ & $0.0126(19)$ & 5.3 & 0.98 & $\mathbf{0 . 0 7 9 6}\left(f_{1}\right)$ & $0.8702(400)$ & 5.3 & 0.99 \\
\hline$f_{52}$ & 0.24531 & $0.000409(114)$ & 2.7 & & $0.4461\left(f_{35}\right)$ & $0.0076(19)$ & 3.6 & & $0.4467\left(f_{35}\right)$ & $0.2861(409)$ & 3.7 & \\
\hline$f_{61}$ & 0.44192 & $0.000355(108)$ & 2.6 & & $0.8488\left(f_{67}\right)$ & $0.0075(20)$ & 3.8 & & $0.8497\left(f_{67}\right)$ & $0.3546(399)$ & 3.4 & \\
\hline$f_{67}$ & 0.85214 & $0.000323(105)$ & 2.8 & & $0.1462\left(f_{7}\right)$ & $0.0071(21)$ & 3.1 & & $0.2425\left(f_{52}\right)$ & $0.4285(456)$ & 3.1 & \\
\hline$f_{99}$ & 0.89541 & $0.000266(88)$ & 2.6 & & & & Mag & esium 1 & e $(\lambda 4481.1 \AA)$ & & & \\
\hline$f_{131}$ & 0.97831 & $0.000241(76)$ & 2.6 & & $0.9829\left(f_{131}\right)$ & $0.0138(12)$ & 3.7 & & $0.0805\left(f_{1}\right)$ & $1.0214(504)$ & 5.9 & 1.00 \\
\hline$f_{165}$ & 0.60610 & $0.000192(65)$ & 2.6 & & $0.0793\left(f_{1}\right)$ & $0.0051(11)$ & 4.9 & 0.99 & $0.4461\left(f_{35}\right)$ & $0.5414(503)$ & 3.8 & \\
\hline$f_{216}$ & 0.98671 & $0.000147(53)$ & 2.5 & & $0.9896\left(f_{216}\right)$ & $0.0045(12)$ & 3.2 & & $1.2163\left(f_{281}\right)$ & $0.5046(522)$ & 3.6 & \\
\hline$f_{281}$ & 1.21676 & $0.000123(43)$ & 2.6 & & $0.4461\left(f_{35}\right)$ & $0.0025(11)$ & 3.0 & & $0.2934\left(f_{25}\right)$ & $0.3932(546)$ & 3.3 & \\
\hline
\end{tabular}




\subsection{Doppler imaging}

The DI technique allows us to invert time series of observations into the stellar surface distribution maps of different physical parameters (elemental abundances, temperatures, magnetic field, etc.). This method is widely used for studying chemically peculiar (CP) stars that show a non-uniform distribution of one or several chemical element abundances on their surfaces.

Though stellar surface inhomogeneities were mainly detected in A- and late B-type as well as in cooler stars so far, we recently started to observe a similar behaviour in more massive and hotter early B-type stars too. Similar to A-type stars, stellar surface inhomogeneities observed in these objects are usually accompanied by strong and, sometimes, complex magnetic fields suggesting that it is this field that is responsible for the formation of spots. For example, Kochukhov et al. (2011) presented the results of the analysis of early B-type CP star HD 37776. The authors reported on the detection of strong and complex magnetic field as well as on the inhomogeneous distribution of $\mathrm{He}$ on the surface of this star. Rivinius et al. (2013) reported on strong LPV of both carbon and helium photospheric lines in the helium strong B star HR 7355. The authors attribute the variability to the surface abundance spots with a rather large, a couple of dex, gradient between the regions of enhancement and depletion of the chemical element. Degroote et al. (2011) and Pápics et al. (2012) reported on the detection of the rotational signal from both $C o R o T$ photometry and high-resolution spectroscopy in two B-type stars HD 174648 and HD 43317, respectively. Degroote et al. (2011) showed that for HD 174648, the signal can be explained by the two circumpolar spots, whereas the spot-like scenario for HD 43317 is supported by the recent detection of a magnetic field for this star (Briquet et al. 2013). Petit et al. (2013) presented a summary of physical, rotational and magnetic properties of all known magnetic O and B stars (see their table 1). Hubrig et al. (2006) reported the detection of a weak longitudinal magnetic field of the order of a few hundred of gauss in 13 Slowly Pulsating B (SPB) stars and one $\beta$ Cep variable ( $\xi^{1} \mathrm{CMa}$ ) although this was recently criticized (Bagnulo et al. 2012; Shultz et al. 2012).

There are several theories to explain the existence of the magnetic fields in massive, early-type stars. The most disseminated theory is the one of fossil magnetic fields, i.e. the remnant of the magnetic field gained by the star in the course of its formation phase (Braithwaite \& Nordlund 2006). There are also theories proposing that the convective core of the massive stars (Brun, Browning \& Toomre 2005) or a dynamo process operating in the differentially rotating radiative layers (Mullan \& MacDonald 2005) is at the origin of the magnetic fields observed at the surfaces of these stars. Cantiello et al. (2009, 2011) suggested, from their theoretical calculations and the magnetohydrodynamic simulations, the presence of a sub-surface convective region in massive stars that is capable of producing localized magnetic fields observable at their surfaces. The authors also suggested that this convective region might be responsible for the large microturbulent velocity fields typical for massive early-type stars.

The fact that Si III line profiles of the primary component of V380 Cyg show a clear variability with the period close to the (expected) rotation period of the star suggests that the system may contain yet another rotationally modulated B-type star. Phase-folded silicon profiles on which we base our further analysis (see below) show the same kind of variability as shown in Fig. 9 (bottom).

We used the Si III spectral line at $\lambda 4452 \AA$ to check whether the observed LPV can be explained by the rotation effect due to spot(s) on the stellar surface. The analysis is based on the above- described method of DI as implemented in the INVERS8 FORTRAN code (Piskunov \& Rice 1993). The code inverts time series of the observed line profiles into surface abundance distribution maps by minimizing the difference between the observations and the model. Since the problem is strongly ill-posed, it has to be regularized which is done by means of Tikhonov regularization. From a mathematical point of view, the regularization is performed by adding an additional term of the form $\Lambda \mathrm{F}(\epsilon)$ to the total error function that is a subject of minimization, where $\Lambda$ represents a regularization parameter (usually, taking a value between $10^{-2}$ and $10^{-4}$ ) and $\mathrm{F}(\epsilon)$ stands for the regularization functional. The algorithm then iteratively reduces the total error function until the discrepancy between the observations and the model is of the same order as the individual errors of measurement (Kochukhov 2003).

Calculations with the INVERS 8 code are based on the pre-computed tables of the local line profiles. These profiles are computed for different positions on the stellar disc (20 in our case), which allows us to take the centre-to-limb variation of the intensity, and for a set of individual abundances of a given chemical element. The profiles are then convolved with the instrumental profile of a certain width, which in our case corresponds to the high-resolution mode $(R=$ 85000 ) of the HERMES instrument (Raskin et al. 2011). Since the primary component of V380 Cyg is a hot B-type giant star, non-LTE line formation must be assumed when synthesizing its line profiles. The local profiles were computed with the non-LTE version of the SYNTHV code developed by VT. To synthesize the spectra, the code uses pre-computed non-LTE departure coefficients, which in our case were calculated with the TLUSTY model atmospheres code (Hubeny 1988; Hubeny \& Lanz 1995).

In our calculations with the INVERS 8 code, we limited the total number of iterations to 500 but in all cases the program converged much faster, typically after completing 170-200 iterations. The calculations were performed using three values of the regularization parameter $\Lambda: 10^{-2}, 10^{-3}$ and $10^{-4}$. The resulting Si-abundance maps for all the three values of $\Lambda$ are shown in Fig. 10 (left) in Mercator projection. Obviously, in all three cases, the algorithm favours a solution with two 'dominant' spots, of which one is bright (abundance depletion) and another is dark (abundance enrichment). The map computed for $\Lambda=10^{-2}$ also exhibits a third spot at a longitude of about $130^{\circ}$ but it is of much lower contrast compared to the bulk of the star than the two others. The essential difference between this map (top) and the two other maps (middle and bottom) is that the spots are located at almost the same latitude $\phi$ but at different longitudes $\theta$ such that only one of the high-contrast spots is visible at a given instant of time (except for the phases when both spots are visible at the very limb of the star). In the two other cases, the spots have rather the same longitude but different latitudes which makes them invisible for almost half of the rotation cycle. The quality of the fit of the observed profiles for all three maps is almost the same, as is illustrated in Fig. 10 (right). The major part of the observed spectroscopic variability can be explained by either of our models, also confirming the suggestion made in Sections 3.2 and 4.4 that the star shows a signature of rotational modulation.

A careful look at the obtained maps and the corresponding abundance gradients allows us to exclude the one computed for the lowest value of $\Lambda=10^{-4}$ (cf. Fig. 10, bottom). This map exhibits an unreliably high gradient of the $\mathrm{Si}$ abundance of about 2 dex between the two spots, which is compensated by other small-scale structures on the stellar surface (e.g. low-contrast but elongated spots at $\left.[\theta, \phi]=\left[-135^{\circ},+55^{\circ}\right] ;\left[-75^{\circ},+0^{\circ}\right]\right)$ to provide a good fit to the observations. Moreover, the rather high mean abundance (without taking the two spots into account) of $\sim 3.5$ dex characteristic of this 

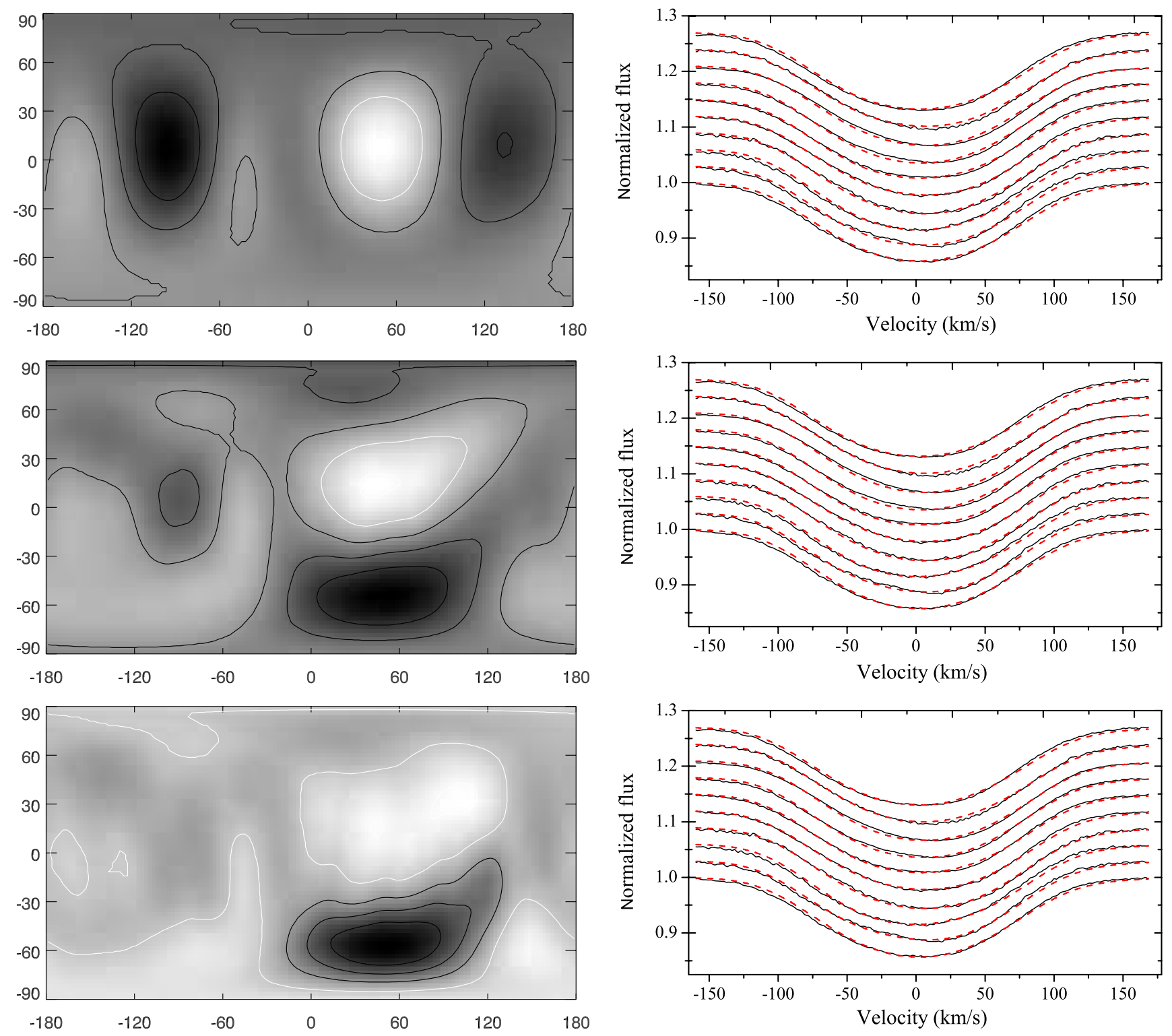

Figure 10. Si-abundance surface maps in Mercator projection (left) and the comparison between the observed (solid black line) and computed (dashed red line) profiles. The calculations are based on three values of the regularization parameter $\Lambda: 10^{-2}, 10^{-3}$ and $10^{-4}$ (from top to bottom). The profiles corresponding to different rotation phases are vertically shifted by a small, constant value for better visibility. $X$ - and $Y$-axes on the maps give longitude $\theta$ and latitude $\phi$ coordinates, respectively. The dark and bright areas refer to the regions of enhanced and depleted Si abundance, respectively. The maximal abundance gradient increases from the top map to the bottom and is (in dex): [Min:Max $]=[-4.41:-4.10]-$ top, $[-4.65:-3.61]-$ middle and $[-4.69:-2.56]-$ bottom.

map is not compatible with the Si abundance estimated from the spectrum analysis of the disentangled spectrum of the primary (cf. Section 4.2). To some extent, this also concerns the map computed for $\Lambda=10^{-3}$ (cf. Fig. 10, middle), as the estimated mean Si abundance for this map is $\sim 3.95$ dex which also seems to be too high. We thus favour the map showing two large spots located at almost the same latitude (cf. Fig. 10, top) though we are aware that it is rather difficult to decide which of the two maps $\left(\Lambda=10^{-2}\right.$ or $\left.10^{-3}\right)$ is the most appropriate one.

Fig. 11 shows the selected map in spherical projection and at four different rotational phases. Though the detected total Si-abundance gradient of about 0.3 dex is small, it is significant compared to the estimated errors. As stated by Piskunov \& Rice (1993), the most reliable errors on the reconstructed abundances are obtained by 'mapping' several spectral lines of the same chemical element separately. We thus performed the DI analysis of all the three $\mathrm{Si}$ lines used by us for the frequency analysis (cf. Table 8). The surface abundance distributions obtained from the individual lines are compatible with each other, also when assuming different values of the regularization parameters $\Lambda$. The delivered abundance gradients are consistent as well, giving a maximum abundance amplitude of $\sim 0.35$ dex for the map computed with $\Lambda=10^{-2}$. The errors estimated from the abundance scatter between the maps obtained based on the three individual spectral lines are of the order of 0.1 dex, thanks to the very high $\mathrm{S} / \mathrm{N}$ of our spectroscopic data, which makes the detection of such a low abundance gradient possible. The continuum normalization of the spectra usually also serves as an additional source of uncertainty on the reconstructed maps, but is minimal in our case due to the high quality of the data. 

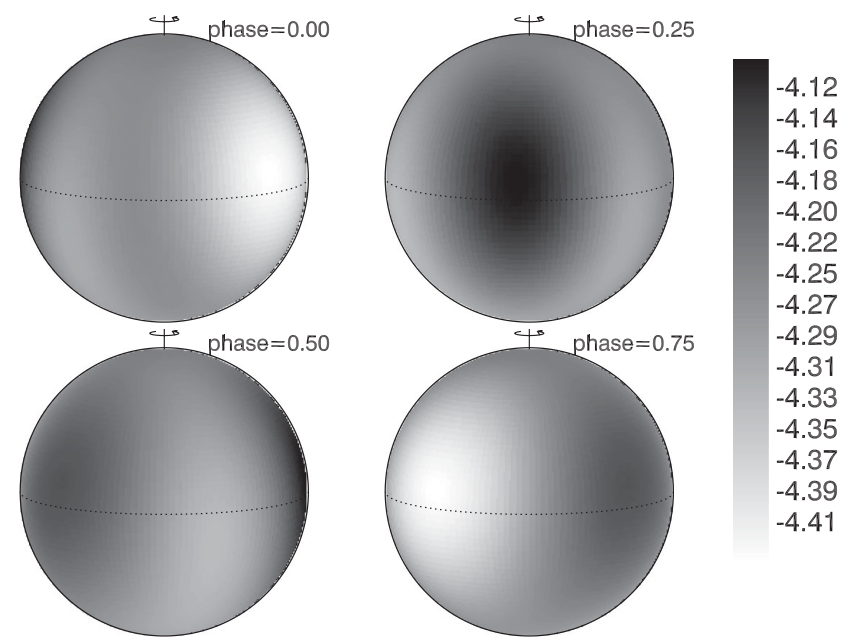

Figure 11. Si-abundance map in spherical projection computed for $\Lambda=$ $10^{-2}$. The dark and bright areas refer to the regions of enhanced and depleted Si abundance, respectively.

As an additional check for the consistency between the frequency analysis of the line profiles and the DI results, we applied the algorithm to both $\mathrm{He}$ and $\mathrm{Mg}$ lines in which we could not detect the signal that we interpret as being due to rotation of the star. Similar to the case of silicon, we used the same spectral lines as for the frequency analysis, i.e. two $\mathrm{He}$ and one $\mathrm{Mg}$ lines (cf. Table 8). It is worth noting that a visual inspection of the lines of both elements folded with the rotation period was enough to conclude that they do not exhibit variability similar to the silicon lines but are stable over the rotation cycle instead. Indeed, the DI algorithm delivers rather structured surface maps even for the regularization parameter of $\Lambda=10^{-2}$ and with a very low abundance gradient below 0.1 dex. Such a gradient is considered insignificant as it is comparable to the estimated uncertainties, and we conclude that both helium and magnesium show a uniform distribution of their abundances over the stellar surface.

\subsection{Evolutionary models}

As already mentioned (cf. Section 1), both components of the V380 Cyg system show a discrepancy between the dynamical mass and the one obtained from evolutionary models. P2009 showed that the discrepancy in mass does not disappear with the inclusion of rotation effects into the evolutionary models, whereas G2000 suggested a high value of the convective overshoot parameter $\left(\alpha_{\mathrm{ov}}=0.6 \pm 0.1\right)$ as a possible solution for the mass problem. In the present study, the fundamental stellar parameters of both components of V380 Cyg are refined, and used to compare to a new set of evolutionary model calculations. We use the MESA stellar evolution code (Paxton et al. 2011, 2013) to compute the tracks. The basic setup includes an initial hydrogen mass fraction of $X=0.70$ and metal abundance fractions from Asplund et al. (2009). The initial rotation velocity on the pre-main-sequence track is set to $v_{\text {init }}=0 \mathrm{~km} \mathrm{~s}^{-1}$ (no rotation), and we choose to include core overshooting as a step function, parametrized with an $\alpha_{\mathrm{ov}}=0.2$ pressure scaleheight by default. The Schwarzschild criterion is used in the convection treatment. We choose a standard mixing length $\alpha_{\text {MLT }}=1.8$ pressure scale-height. The MESA equation of state is used, and the $\mathrm{N}^{14}$ reaction rate from Imbriani et al. (2004). We compared the stellar models computed with MESA to those in the grid of stellar models computed with the Code Liégois d'Évolution
Stellaire (CLES) by Briquet et al. (2011) and used by Saesen et al. (2013) for asteroseismic modelling of B-type pulsators in the open cluster NGC 884. Excellent agreement was found for the same input physics.

Figs 12-14 show the location of both components of V380 Cyg in the $T_{\text {eff }}-\log g$ diagram along with the evolutionary tracks. Fig. 12 illustrates the influence of mass and bulk metallicity on the theoretical tracks. One can see that both parameters have a similar effect on the models, namely a mass increase or metallicity decrease shifts the tracks to the left in the $T_{\text {eff }}-\log g$ diagram, i.e. towards higher effective temperatures. The effects of core convective overshooting and of the initial rotational velocity are shown in Fig. 13. We see that both phenomena increase the stellar lifetime on the main sequence, with the effect being larger for the overshooting.

From Fig. 12 (left-hand panel), it is clear that a discrepancy of about $0.85 \mathrm{M}_{\odot}$ between the dynamical and theoretical masses exists for the secondary component. The right-hand panel of Fig. 12 shows that one can match these two masses by assuming a lower bulk metallicity for the $\operatorname{star}(Z=0.006)$. The model calculations represent the case of a non-rotating star and assume a value of $\alpha_{\mathrm{ov}}=0.2$. A non-rotating model appears to be a reasonable approximation for the secondary since its estimated rotation rate is $8.0 \pm 0.8$ percent of the critical velocity. However, the assumed low bulk metallicity for the secondary is not consistent with the analysis of its disentangled spectrum, which showed close-to-solar atmospheric abundances (cf. Section 4.2). The two models that fit the position of the star in the $T_{\text {eff }}-\log g$ diagram, taking into account the error bars, are illustrated in Fig. 14 by long- and short-dashed lines (hereafter models 2 and 3, respectively). Both models assume the same mass of $7.42 \mathrm{M}_{\odot}$ (within $3 \sigma$ from the dynamical mass derived in Section 3.1), chemical composition $Z=0.012$ dex and no rotation, but a significantly different overshooting parameter of $\alpha_{\text {ov }}=0.6$ and 0.2 for models 2 and 3 , respectively. The estimated age of the star from these two models is $21.5 \pm 1.5$ and $18 \pm 1 \mathrm{Myr}$, respectively.

Assuming that both components of V380 Cyg have the same (initial) metal content $(Z=0.012)$ and age, and that the primary could be either in the core or shell hydrogen burning phase of the evolution (models 2 and 3 in Table 9, respectively), we attempted to fit its position in the $T_{\text {eff }}-\log g$ diagram. As we can see in Fig. 14, for model 2 to match the observations, we need to introduce an unreasonably high rotation $\left(v_{\text {ZAMS }}=240 \mathrm{~km} \mathrm{~s}^{-1}\right.$ which corresponds to the rotational velocity of $330 \mathrm{~km} \mathrm{~s}^{-1}$ at the location of the star in Fig. 14), an extremely strong amount of overshooting $\left(\alpha_{\mathrm{ov}}=0.6\right)$ and a mass at the $3 \sigma \operatorname{limit}\left(M=12.00 \mathrm{M}_{\odot}\right)$. For comparison with the models, we choose the error bars on the effective temperature of the primary to be at the $3 \sigma$ level of the spectroscopic estimate of $300 \mathrm{~K}$ (cf. Table 5). The latter value is the standard deviation of the mean which hardly represents realistic errors (cf. Section 4.2). We find the uncertainty of $900 \mathrm{~K}$ to be more conservative, which allows us (at least partly) to take into account the effects of non-uniform temperature distribution on the surface of the primary due to its nonspherical shape (the star rotates at $\sim 32$ per cent of its critical velocity), imperfect continuum normalization and atomic data, etc. We also show model 3 that allows us to fit the position of the primary in the $T_{\text {eff }}-\log g$ diagram assuming that the star is in the shell hydrogen burning phase (short-dashed line in Fig. 14). Although this model assumes $\alpha_{\mathrm{ov}}=0.3$, we still had to introduce an unreasonably high rotation $\left(v_{\text {ZAMS }}=243 \mathrm{~km} \mathrm{~s}^{-1}\right.$ which corresponds to the rotational velocity of $420 \mathrm{~km} \mathrm{~s}^{-1}$ at the location of the star in the diagram) as well as to increase mass of the star by $\sim 1.5 \mathrm{M}_{\odot}$ compared to the dynamical value. Increasing the mass of the primary enables us to 

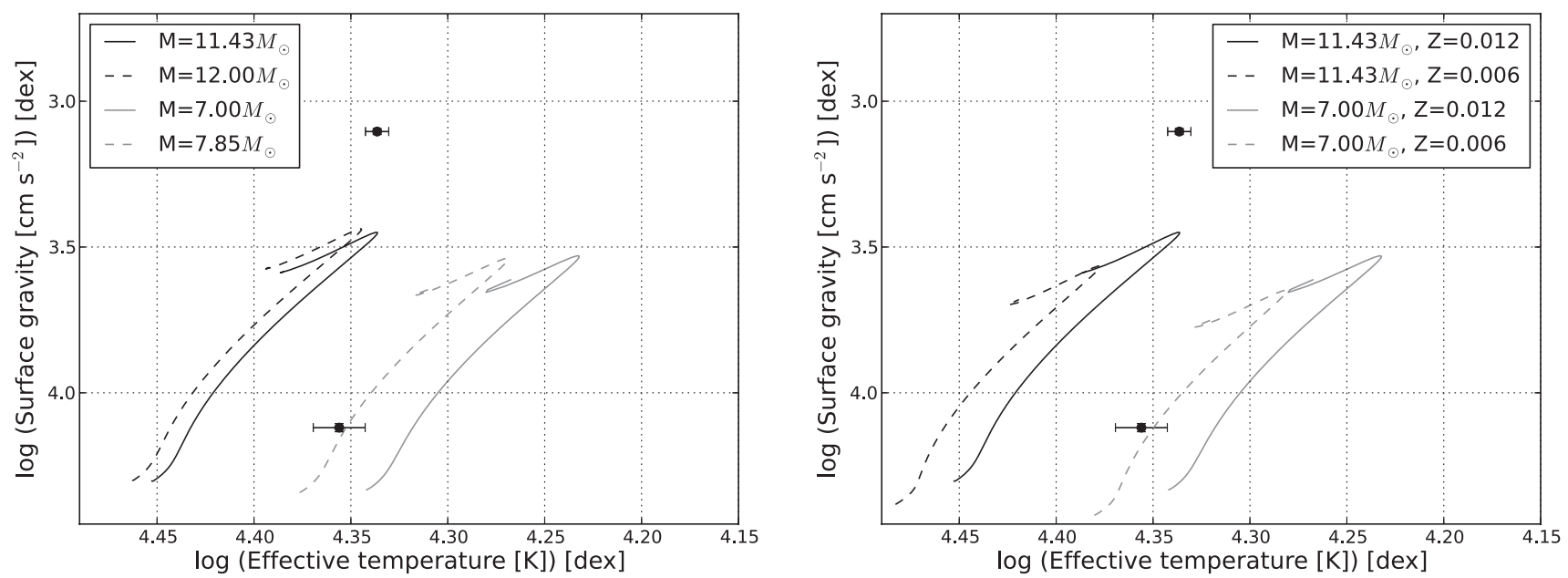

Figure 12. Location of the primary and secondary components of V380 Cyg in the $T_{\text {eff }}-\log g$ diagram. Theoretical evolutionary tracks show the influence of mass (left) and metallicity (right).
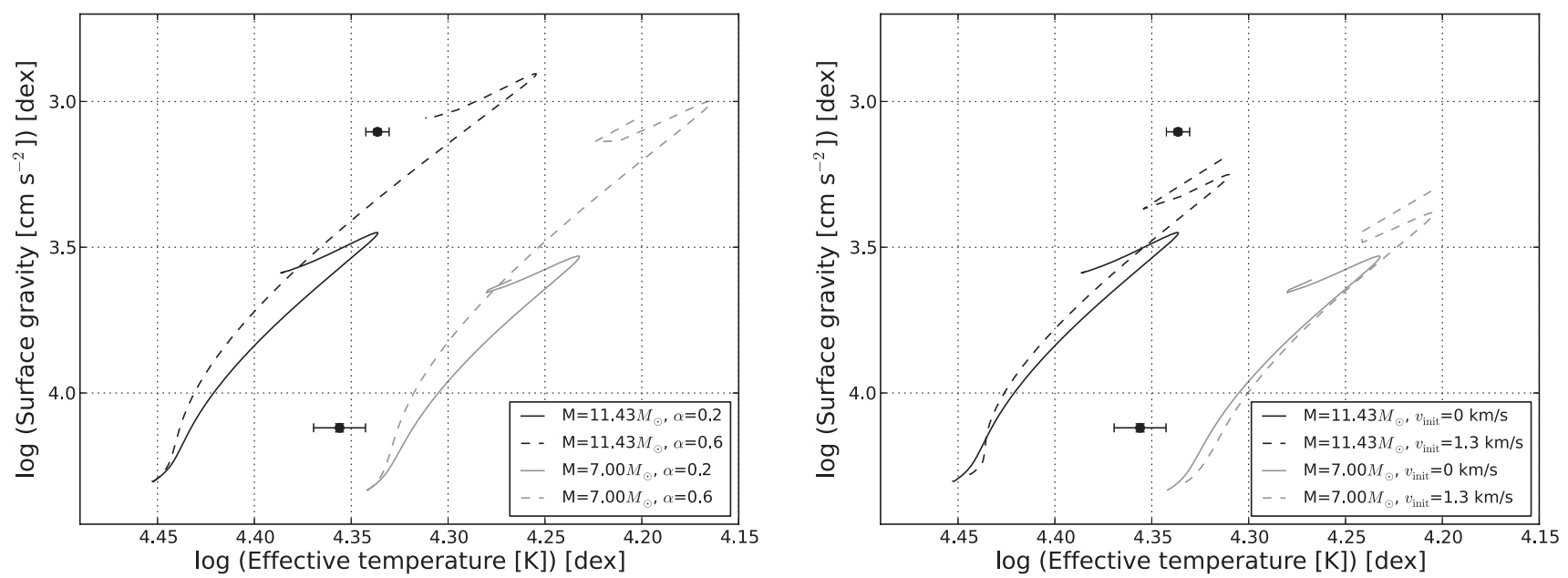

Figure 13. Same as Fig. 12 but for the influence of overshooting (left) and initial rotational velocity (right).

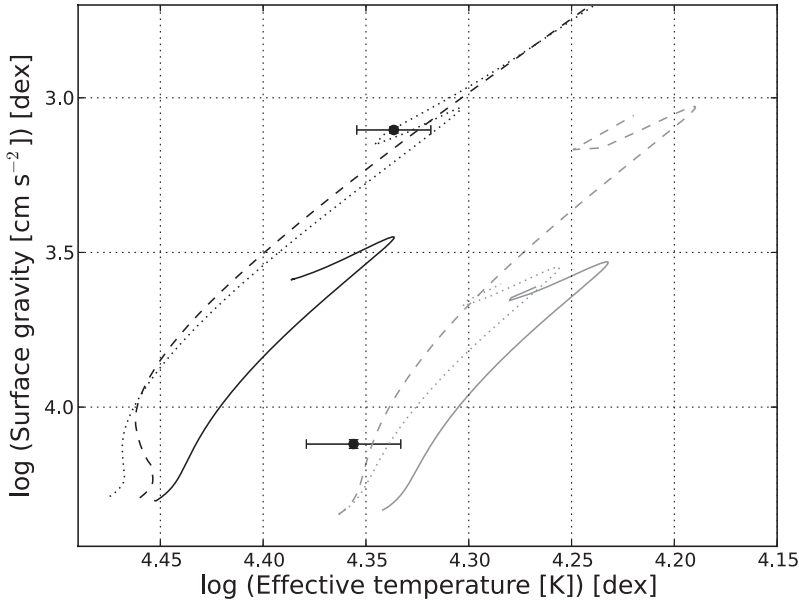

Figure 14. Location of the primary and secondary components of V380 Cyg in the $T_{\text {eff }}-\log g$ diagram. Solid, long- and short-dashed tracks correspond to model 1, 2 and 3 in Table 9, respectively. match the observed location of the primary with track prediction, but it is not reconcilable with the RV measurements. The MESA model predictions clearly point to mass discrepancy for the primary component, in agreement with the findings by G2000 and P2009.

\section{DISCUSSION AND CONCLUSIONS}

In this paper, we presented a detailed study of V380 Cyg, an eccentric binary ( $e=0.22$ ) with two massive components (11.4 and $7.0 \mathrm{M}_{\odot}$ for the primary and secondary, respectively) of spectral type B. We based our analysis on high-precision photometric data gathered with the Kepler space-based telescope, as well as on groundbased high-dispersion spectroscopy obtained with the HERMES spectrograph (Raskin et al. 2011). The data were analysed using state-of-the-art methods to determine orbital parameters of the system and absolute dimensions of both stellar components, as well as to explain both photometric and spectroscopic variability intrinsic to the primary star and discovered only recently by Paper I.

The effects of binarity in the light curve were modelled using the WD code (Wilson \& Devinney 1971; Wilson 1979), while the SPD 
Table 9. Evolutionary model parameters for both components of V380 Cyg.

\begin{tabular}{|c|c|c|c|c|c|c|c|}
\hline \multirow[t]{2}{*}{ Par } & \multirow[t]{2}{*}{ Unit } & \multicolumn{3}{|c|}{ Primary } & \multicolumn{3}{|c|}{ Secondary } \\
\hline & & Model 1 & Model 2 & Model 3 & Model 1 & Model 2 & Model 3 \\
\hline$M$ & $\mathrm{M}_{\odot}$ & 11.43 & 12.00 & 12.90 & 7.00 & 7.42 & 7.42 \\
\hline$Z$ & dex & 0.014 & 0.012 & 0.012 & 0.014 & 0.012 & 0.012 \\
\hline$\alpha_{\mathrm{ov}}$ & $\mathrm{Hp}$ & 0.2 & 0.6 & 0.3 & 0.2 & 0.6 & 0.2 \\
\hline$v$ & $\mathrm{~km} \mathrm{~s}^{-1}$ & 0 & 241 & 243 & 0 & 0 & 0 \\
\hline Age & Myr & - & 21.5 & 18 & - & 21.5 & 18 \\
\hline
\end{tabular}

technique was used to derive the spectroscopic orbit as well as the disentangled spectra of both stellar components. The determined orbital and physical parameters agree well with those reported by G2000 and Paper I. However, we find a considerable difference between the temperature of the secondary obtained from the lightcurve fitting $\left(T_{\text {eff, в }}=23840 \pm 500 \mathrm{~K}\right)$ and the one deduced from the spectral characteristics of this star $\left(T_{\text {eff, B }}=21800 \pm 400 \mathrm{~K}\right)$. We find a similar chemical composition for both binary components and good agreement with the abundances reported by P2009 for the primary component of V380 Cyg.

After subtracting the orbital signal from both the photometric and spectroscopic data, the residuals were subjected to frequency analysis. The results of the photometric and spectroscopic analyses are consistent with each other in the sense that the variability occurs at the orbital frequency and its harmonics as well as at the rotation frequency of the primary. All other variability detected in the $\mathrm{Ke}$ pler data is of stochastic nature. We find that the characteristics of this variability are not in contradiction with the recent theoretical predictions by Cantiello et al. (2009) and Shiode et al. (2013) for the g-mode oscillations excited both in the core and in a thin convective sub-surface layer of massive stars.

We also explored the hypothesis of observing the rotationally modulated signal in our data in more detail. We used the DI technique to study variability detected in several spectral lines of doubly ionized silicon. Two solutions that provide nearly the same quality of the fit but different spot configurations on the stellar surface have been obtained. We favour the one that shows a lower abundance gradient and assumes two high- and one low-contrast spot located at (nearly) the same latitude but at different longitudes.

We used the MESA stellar evolution code to look at the model predictions for both components of V380 Cyg. For the secondary, we found a discrepancy of $\sim 0.85 \mathrm{M}_{\odot}$ between the dynamical and theoretically predicted masses assuming standard stellar evolutionary physics. However, a slightly larger value of the mass (within $3 \sigma$ from the dynamical value) and a bulk metallicity of $Z=0.012$ dex can account for this difference. Two models that fit the position of the primary in the $T_{\text {eff }}-\log g$ diagram were found and provide significantly different age estimates for the system: $21.5 \pm 1.5$ and $18 \pm 1 \mathrm{Myr}$ (cf. Table 9). In both cases, the system appears to be much younger than $25.5 \pm 1.5 \mathrm{Myr}$, the age determined by G2000 assuming $\alpha_{\mathrm{ov}}=0.6$ for the primary component. Since both models assume extreme input physics like a very high rotation at the zeroage main sequence (ZAMS) and/or a large amount of overshooting, we conclude that single-star evolutionary models are not suitable for this particular binary system.

The variability detected in the Kepler data is intrinsic to the primary component and of stochastic nature. The signal is variable both in amplitude and in appearance on a short time-scale. This makes the tuning of the convective core overshooting parameter $\alpha_{\mathrm{ov}}$ from asteroseismic analysis impossible for this star. In future papers, we plan to take a closer look at another massive binary system, Spica, with the aim to see whether the above-mentioned mass discrepancy holds for this star too and whether asteroseismology can help us to constrain the $\alpha_{\text {ov }}$ parameter in that case.

\section{ACKNOWLEDGEMENTS}

The research leading to these results received funding from the European Research Council under the European Community's Seventh Framework Programmes (FP7/2007-2013)/ERC grant agreement no. 227224 (PROSPERITY) and FP7-SPACE-2011-1, project number 312844 (SPACEINN). This research is (partially) funded by the Research Council of the KU Leuven under grant agreement GOA/2013/012.

\section{REFERENCES}

Aerts C., de Pauw M., Waelkens C., 1992, A\&A, 266, 294

Aerts C., Thoul A., Daszyńska J., Scuflaire R., Waelkens C., Dupret M. A., Niemczura E., Noels A., 2003, Science, 300, 1926

Aerts C., Briquet M., Degroote P., Thoul A., van Hoolst T., 2011, A\&A, 534, A98

Asplund M., Grevesse N., Sauval A. J., Scott P., 2009, ARA\&A, 47, 481

Auvergne M. et al., 2009, A\&A, 506, 411

Bagnulo S., Landstreet J. D., Fossati L., Kochukhov O., 2012, A\&A, 538, A129

Batten A. H., 1962, Publ. Dom. Astrophys. Obs. Victoria, 12, 91

Bessell M. S., Castelli F., Plez B., 1998, A\&A, 333, 231

Blomme R. et al., 2011, A\&A, 533, A4

Braithwaite J., Nordlund Å., 2006, A\&A, 450, 1077

Breger M. et al., 1993, A\&A, 271, 482

Briquet M., Morel T., Thoul A., Scuflaire R., Miglio A., Montalbán J., Dupret M.-A., Aerts C., 2007, MNRAS, 381, 1482

Briquet M. et al., 2011, A\&A, 527, A112

Briquet M., Neiner C., Leroy B., Pápics P. I., 2013, A\&A, 557, L16

Brun A. S., Browning M. K., Toomre J., 2005, ApJ, 629, 461

Bryson S. T. et al., 2010, ApJ, 713, L97

Butler K., 1984, PhD thesis, Univ. London

Cantiello M. et al., 2009, A\&A, 499, 279

Cantiello M., Braithwaite J., Brandenburg A., Del Sordo F., Käpylä P., Langer N., 2011, Proc. IAU Symp. 272, Active OB Stars: Structure, Evolution, Mass Loss, and Critical Limits. Cambridge Univ. Press, Cambridge, p. 32

Charbonneau P., 1995, ApJS, 101, 309

Claret A., 2007, A\&A, 475, 1019

Clausen J. V., Torres G., Bruntt H., Andersen J., Nordström B., Stefanik R. P., Latham D. W., Southworth J., 2008, A\&A, 487, 1095

Degroote P. et al., 2009, A\&A, 506, 471

Degroote P. et al., 2011, A\&A, 536, A82

Frémat Y., Lampens P., Hensberge H., 2005, MNRAS, 356, 545

Giddings J. R., 1981, PhD thesis, Univ. London

Guinan E. F., Ribas I., Fitzpatrick E. L., Giménez Á., Jordi C., McCook G. P., Popper D. M., 2000, ApJ, 544, 409 (G2000)

Hadrava P., 1995, A\&AS, 114, 393

Hareter M. et al., 2008, A\&A, 492, 185 
Hensberge H., Pavlovski K., Verschueren W., 2000, A\&A, 358, 553

Hensberge H., Ilijić S., Torres K. B. V., 2008, A\&A, 482, 1031

Herrero A., Kudritzki R. P., Vilchez J. M., Kunze D., Butler K., Haser S., 1992, A\&A, 261, 209

Hilditch R. W., 2004, in Hilditch R. W., Hensberge H., Pavlovski K., eds, ASP Conf. Ser. Vol. 318, Spectroscopically and Spatially Resolving the Components of the Close Binary Stars. Astron. Soc. Pac., San Francisco, p. 198

Hill G., Batten A. H., 1984, A\&A, 141, 39

Hubeny I., 1988, Comput. Phys. Commun., 52, 103

Hubeny I., Lanz T., 1995, ApJ, 439, 875

Hubrig S., Briquet M., Schöller M., De Cat P., Mathys G., Aerts C., 2006, MNRAS, 369, L61

Ilijic S., Hensberge H., Pavlovski K., Freyhammer L. M., 2004, in Hilditch R. W., Hensberge H., Pavlovski K., eds, ASP Conf. Ser. Vol.318, Spectroscopically and Spatially Resolving the Components of the Close Binary Stars. Astron. Soc. Pac., San Francisco, p. 111

Imbriani G. et al., 2004, A\&A, 420, 625

Kochukhov O., 2003, PhD thesis, Uppsala University

Kochukhov O., Lundin A., Romanyuk I., Kudryavtsev D., 2011, ApJ, 726, 24

Kolenberg K. et al., 2011, MNRAS, 411, 878

Kurucz R., 1993, ATLAS9 Stellar Atmosphere Programs and $2 \mathrm{~km} / \mathrm{s}$ grid. Kurucz CD-ROM No. 13. Smithsonian Astrophysical Observatory, Cambridge, MA

Lefever K., Puls J., Morel T., Aerts C., Decin L., Briquet M., 2010, A\&A, 515, A74

Lomb N. R., 1976, Ap\&SS, 39, 447

Lyubimkov L. S., Rachkovskaya T. M., Rostopchin S. I., Tarasov A. E., 1996, Astron. Rep., 40, 46

Lyubimkov L. S., Lambert D. L., Poklad D. B., Rachkovskaya T. M., Rostopchin S. I., 2013, MNRAS, 428, 3497

Mayer P., Harmanec P., Pavlovski K., 2013, A\&A, 550, A2

McLaughlin D. B., 1924, ApJ, 60, 22

Morel T., 2009, Commun. Asteroseismol., 158, 122

Mullan D. J., MacDonald J., 2005, MNRAS, 356, 1139

Neiner C. et al., 2012, A\&A, 546, A47

Nieva M. F., Przybilla N., 2007, A\&A, 467, 295

Nieva M.-F., Przybilla N., 2012, A\&A, 539, A143

Pápics P. I. et al., 2012, A\&A, 542, A55

Pavlovski K., Hensberge H., 2005, A\&A, 439, 309

Pavlovski K., Hensberge H., 2010, in Prša A., Zejda M., eds, ASP Conf. Ser. Vol. 435, Binaries - Key to Comprehension of the Universe. Astron. Soc. Pac., San Francisco, p. 207
Pavlovski K., Southworth J., 2009, MNRAS, 394, 1519

Pavlovski K., Tamajo E., Koubský P., Southworth J., Yang S., Kolbas V., 2009, MNRAS, 400, 791 (P2009)

Paxton B., Bildsten L., Dotter A., Herwig F., Lesaffre P., Timmes F., 2011, ApJS, 192, 3

Paxton B. et al., 2013, ApJS, 208, 4

Petit V. et al., 2013, MNRAS, 429, 398

Piskunov N. E., Rice J. B., 1993, PASP, 105, 1415

Popper D. M., 1981, ApJS, 47, 339

Popper D. M., Guinan E. F., 1998, PASP, 110, 572

Puls J., Vink J. S., Najarro F., 2008, A\&AR, 16, 209

Raskin G. et al., 2011, A\&A, 526, A69

Rivinius T., Townsend R. H. D., Kochukhov O., Štefl S., Baade D., Barrera L., Szeifert Th., 2013, MNRAS, 429, 177

Rogers T. M., Lin D. N. C., McElwaine J. N., Lau H. H. B., 2013, ApJ, 772, 21

Rossiter R. A., 1924, ApJ, 60, 15

Saesen S., Briquet M., Aerts C., Miglio A., Carrier F., 2013, AJ, 146, 102

Scargle J. D., 1982, ApJ, 263, 835

Schwarzschild M., 1975, ApJ, 195, 137

Shiode J. H., Quataert E., Cantiello M., Bildsten L., 2013, MNRAS, 430, 1736

Shultz M. et al., 2012, ApJ, 750, 2

Simon K. P., Sturm E., 1994, A\&A, 281, 286

Southworth J., Maxted P. F. L., Smalley B., 2005, A\&A, 429, 645

Southworth J. et al., 2011, MNRAS, 414, 2413

Tkachenko A., Lehmann H., Mkrtichian D. E., 2009, A\&A, 504, 991

Tkachenko A. et al., 2012, MNRAS, 424, L21 (Paper I)

van Hamme W., 1993, AJ, 106, 2096

Wilson R. E., 1979, ApJ, 234, 1054

Wilson R. E., 1993, in Leung K.-C., Nha I.-S., eds, ASP Conf. Ser. Vol. 38, New Frontiers in Binary Star Research. Astron. Soc. Pac., San Francisco, p. 91

Wilson R. E., Devinney E. J., 1971, ApJ, 166, 605

Wilson R. E., Van Hamme W., 2004, Computing Binary Star Observables (Wilson-Devinney program user guide). Univ. Florida, Gainsville, Florida

Zima W., 2008, Commun. Asteroseismol., 155, 17

This paper has been typeset from a $\mathrm{T}_{\mathrm{E}} \mathrm{X} / \mathrm{L} \mathrm{T}_{\mathrm{E}} \mathrm{X}$ file prepared by the author. 